ARTICLE

DOI: $10.1038 / s 41467-018-04774-9$

\title{
mTORC1 accelerates retinal development via the immunoproteasome
}

Ji-Heon Choi ${ }^{1}$, Hong Seok Jo1,2, Soyeon Lim¹, Hyoung-Tai Kim¹, Kang Woo Lee ${ }^{1}$, Kyeong Hwan Moon, Taejeong Ha${ }^{1}$, Sang Soo Kwak ${ }^{1}$, Yeha Kim', Eun Jung Lee ${ }^{1}$, Cheol O. Joe ${ }^{1}$ \& Jin Woo Kim (1) ${ }^{1}$

The numbers and types of cells constituting vertebrate neural tissues are determined by cellular mechanisms that couple neurogenesis to the proliferation of neural progenitor cells. Here we identified a role of mammalian target of rapamycin complex 1 (mTORC1) in the development of neural tissue, showing that it accelerates progenitor cell cycle progression and neurogenesis in mTORC1-hyperactive tuberous sclerosis complex 1 (Tsc1)-deficient mouse retina. We also show that concomitant loss of immunoproteasome subunit Psmb9, which is induced by Stat1 (signal transducer and activator of transcription factor 1), decelerates cell cycle progression of Tsc1-deficient mouse retinal progenitor cells and normalizes retinal developmental schedule. Collectively, our results establish a developmental role for $\mathrm{mTORC1}$, showing that it promotes neural development through activation of protein turnover via a mechanism involving the immunoproteasome.

\footnotetext{
${ }^{1}$ Department of Biological Sciences, Korea Advanced Institute of Science and Technology (KAIST), Daejeon 34141, South Korea. ${ }^{2}$ Present address: MEDIMABBIO INC., Seoul 06306, South Korea. These authors contributed equally: Ji-Heon Choi, Hong Seok Jo. Correspondence and requests for materials should be addressed to J.W.K. (email: jinwookim@kaist.ac.kr)
} 
ells constituting the vertebrate nervous system are derived from neural progenitor cells (NPCs), which repeatedly divide to self-renew and produce various types of neurons and glia in particular developmental schedules ${ }^{1,2}$. The proliferating NPC population decreases gradually during development, while post-mitotic neurons (PMNs) derived from the NPCs a
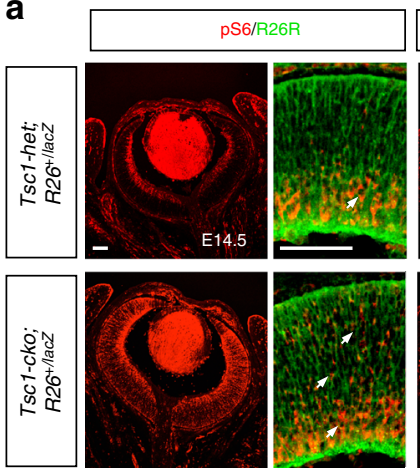

d

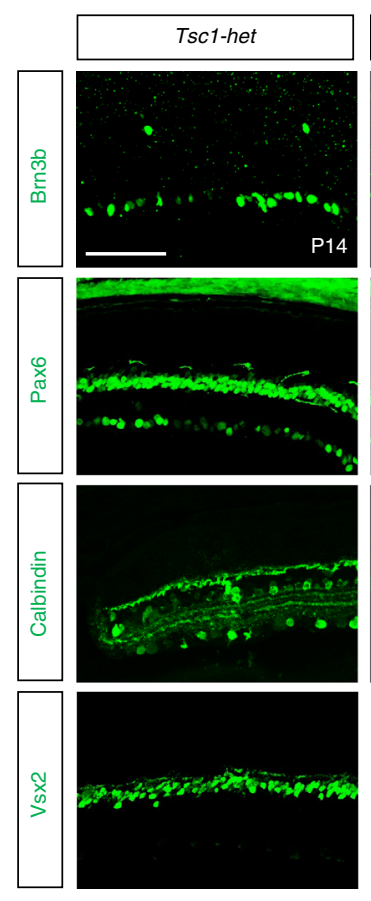

f

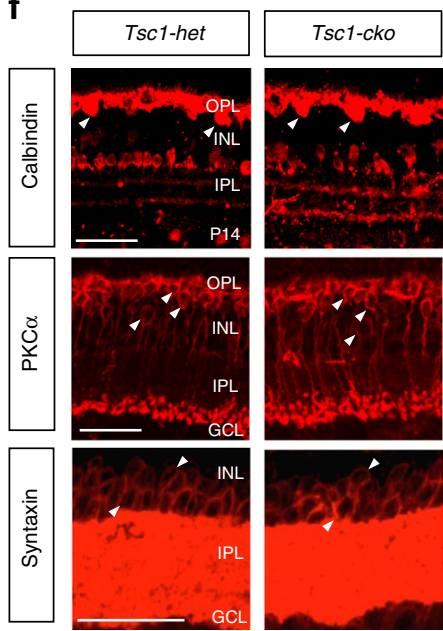

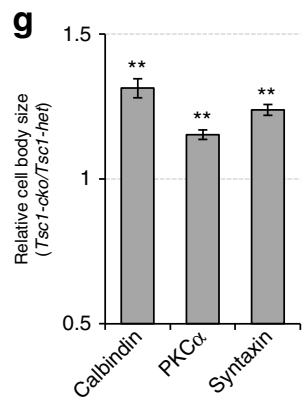

accumulate in neural tissues ${ }^{3}$. These changes not only result from a decrease in the proliferating NPC population within the tissues but also reflect slow progression of the progenitor cell cycle ${ }^{3}$. Therefore, alterations of cell cycle regulator expression often result in the changes in size and composition of neural tissues. For instance, the mice lacking cyclin D1 (CcnD1), which is b

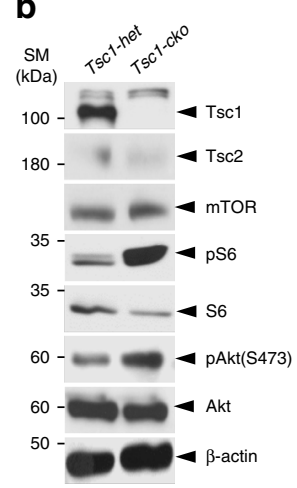

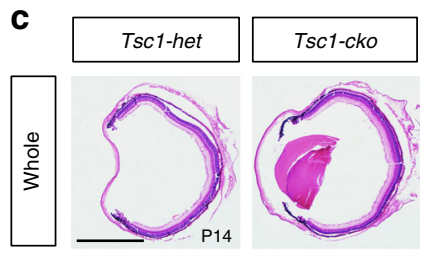
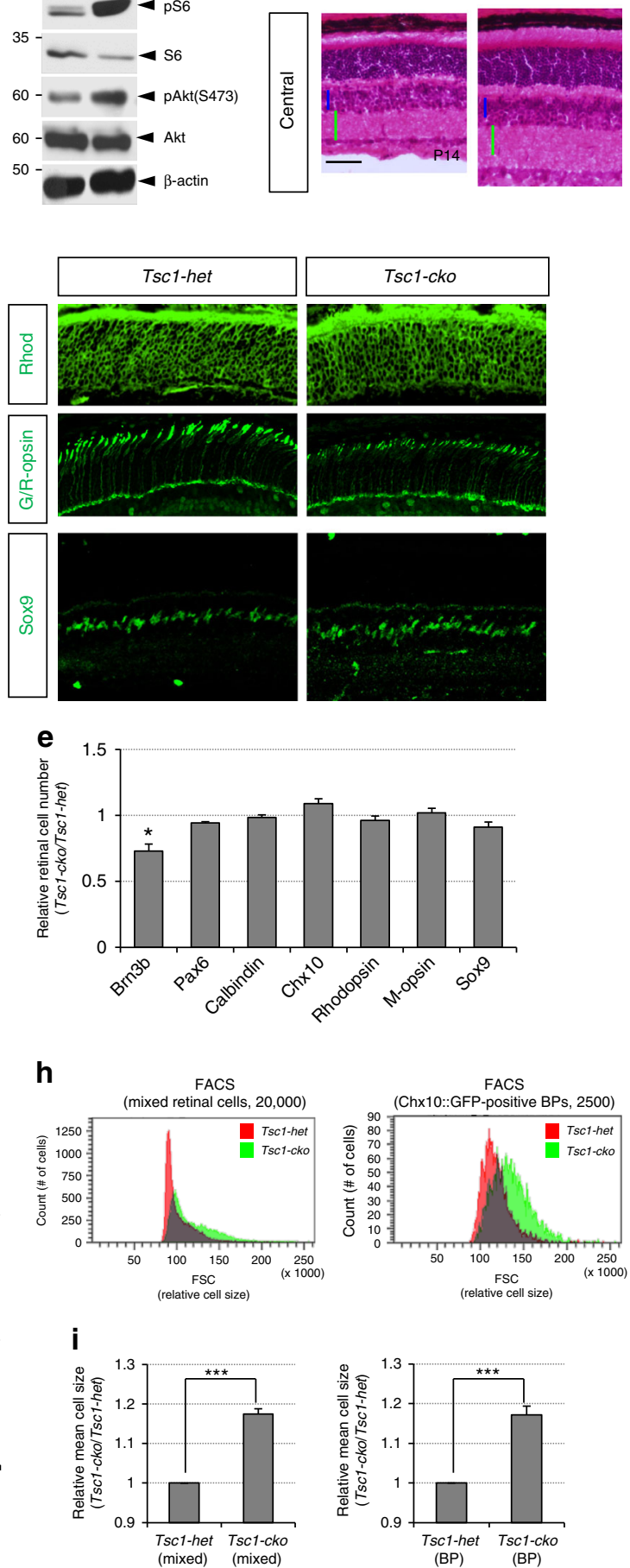
necessary for NPC to stay in cell cycle, show reduced body and organ sizes ${ }^{4}$. The numbers of cells comprising of $C c n D 1$-deficient mouse retina were also remarkably reduced, more significantly in early-born cell types, and this was accounted by slow cell cycle progression and fast cell cycle exit of $C c n D 1$-deficient $\mathrm{NPCs}^{5}{ }^{5}$. Concomitant loss of $p 27^{K i p 1}$ rescues histogenic defects in the $C c n D 1$-deficient mouse retina by preventing $C c n D 1$-deficient NPCs from exiting the cell cycle precociously ${ }^{7}$. Therefore, not only keeping NPC population in proper numbers but also maintaining NPC cell cycle at proper speed is necessary to construct nervous tissues with constant size and composition.

The mouse retina has been employed as a model for studying developmental mechanisms shared among mammalian nervous tissues ${ }^{8}$. Previous studies have found that retinal progenitor cells (RPCs) undergo a serial transition of cell fate during development, producing six major neuronal subtypes and Müller glia (MG) in a stage-specific manner ${ }^{1,8}$. The transition is not only related to serial changes in fate determinants in RPCs; it is also linked to the changes of environment established by newly produced retinal neurons ${ }^{1,9}$. The contribution of internal determinants gradually decreases, whereas external factors produced by neighboring neurons come to play greater roles over time. For instance, growth differentiation factor 11 (Gdf11) is produced by retinal ganglion cells (RGCs) and instructs neighboring RPCs to stop producing additional $\mathrm{RGCs}^{10}$. Sonic hedgehog is also produced by RGCs, but it maintains RPC fate ${ }^{11}$. Therefore, decisions of RPCs to maintain their fate or exit cell cycle to differentiate to retinal neurons are combined outputs of internal determinants and external signals.

The external factors trigger their unique intracellular signaling cascades, which control the fates of RPCs in an evolutionarily conserved manner ${ }^{8,12}$. At the same time, these signals are also integrated into intracellular signaling pathways that are ubiquitously distributed and regulate cell proliferation, differentiation, and survival. The phosphoinositide 3-kinase (PI3K)-Akt signaling cascade is one of the ubiquitous intracellular signaling pathways that couple retinal neurogenesis and RPC proliferation in diverse organisms $s^{13}, 14$. Drosophila eye imaginal disc cells that either overexpress the PI3K catalytic subunit, p110, or lack the phosphoinositide 3-phosphatase phosphatase tensin homolog (PTEN), hyperproliferate to produce more retinal neurons than neighboring wild-type (WT) eye disc cells ${ }^{13}$. Similar autonomous neurogenic acceleration has also been observed in Pten-deficient mouse retinas, in which hyperproliferating RPCs produce all types of retinal neurons in advance of their regular schedule ${ }^{14}$. In both cases, Akt-hyperactive RPCs do not simply expand themselves, but they also produce retinal neurons more rapidly than neighboring WT RPCs.
However, the Akt downstream targets responsible for the diverse changes in the retinas, including hyperproliferation, accelerated neuronal differentiation, enhanced survival, and synaptic dynamics of retinal neurons, remain unclear ${ }^{13-16}$. Among downstream components of PI3K-Akt pathway, the target of rapamycin (TOR) is an evolutionarily conserved serine/ threonine protein kinase that interacts with two distinct sets of adaptor proteins to form the TOR complex 1 (TORC1) and complex 2 (TORC2). The mammalian TORC1 (mTORC1) regulates cell proliferation and growth, while mTORC2 mainly involve in cell shape change and motility ${ }^{17}$. Akt phosphorylates and inactivates tuberous sclerosis complex 2 (TSC2), the GTPaseactivating protein that inhibits Ras homolog enriched in brain 1 (RHEB1) GTPase and thereby interferes with RHEB1-dependent activation of TORC1 ${ }^{18,19}$. The mTORC1 not only supports cell proliferation at downstream of PI3K-Akt pathway but it also senses cellular energy levels to facilitate anabolic processes, such as protein and lipid synthesis, but limits catabolic processes, such as autophagy and lysosome biogenesis ${ }^{17}, 20$. Hyperactivation of mTORC1 either by activating mutations of mTORC1 components or inactivating mutations of TSC closely related with various neurological diseases, such as brain tumors, epilepsy, and autism $^{21}$. The evidences also demonstrate that TORC1 supports neurogenesis in the retina of Drosophila and zebrafish ${ }^{13,22}$.

In this study, we investigate the roles of mTORC1 as a downstream mediator of Akt-induced developmental changes in mouse retina. In tuberous sclerosis complex 1 (Tscl)-deficient mouse retina where mTORC1 is hyperactive, all retinal cells were born ahead of their regular developmental schedules. This was related with the mTORC1-dependent acceleration of progenitor cell cycle. To expedite cell cycle progression, mTORC1 not only facilitates synthesis but also promote degradation of the proteins, including cyclins. For the latter event, mTORC1 induces the expression of immunoproteasome component Psmb9 via signal transducer and activator of transcription 1 (Statl) in the RPC. Collectively, our results suggest a role of the PI3K-Akt-mTORC1 pathway, which promotes the development of neural tissues by coupling synthesis and degradation of cell cycle regulator proteins in the progenitor cells.

\section{Results}

Developmental acceleration of the Tsc1-cko mouse retina. Given the hyperactivation of mTOR in the Akt-hyperactive Ptencko mouse retina (Supplementary Fig. 1), we hypothesized that mTOR pathway might play a role in the PI3K-Akt-induced developmental acceleration of the mouse retina as it regulates Drosophila retinal neurogenesis ${ }^{13}$. To test this hypothesis, we

Fig. 1 Normal cell composition but neuronal enlargement of Tsc1-cko mouse retina. a Distribution of cells underwent Cre-mediated deletion of Tsc1 gene in E14.5 Tsc1-cko;R26 ${ }^{+/ l a c Z}$ mouse retina was visualized indirectly by immunodetection of B-galactosidase (B-gal), which is expressed from a lacZ gene at Crerecombined $R 26 R$ locus. Activities of $\mathrm{mTORC1}$ and $\mathrm{mTORC} 2$ in the retinas were also measured by immunodetection of pS6 and pAkt(S473), respectively. Scale bars, $100 \mu \mathrm{m}$. b Relative levels of mTOR pathway components in the mouse retinas were examined by western blotting (WB) with antibodies against corresponding proteins. SM size marker. c Hematoxylin and eosin (H\&E) staining images of P14 Tsc1-het and Tsc1-cko littermate mouse retinal sections. Sizes of blue and green bars in two bottom images are same. Scale bars, $100 \mu \mathrm{m}$. d P14 littermate mouse eye sections were stained with antibodies that recognize Brn3b (RGC), Pax6 (AC), Calbindin (AC subset and HZ [arrowheads]), Chx10 (BP), Rhodopsin (Rhod; rPR), green/red-opsin (G/R-opsin; cPR), and Sox9 (MG). Scale bars, $200 \mu \mathrm{m}$. e Relative numbers of cells expressing the markers in the Tsc1-cko retinas were obtained by comparing with those in

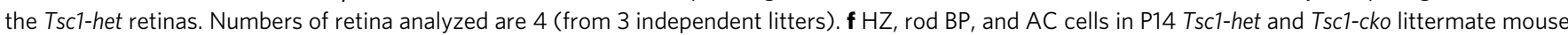
retinas are visualized by immunostainings with antibodies detecting respective markers Calbindin, protein kinase C- $\alpha$ (PKC $\alpha$ ), and Syntaxin. Arrowheads indicate cell bodies of those retinal neurons. $\mathbf{g}$ Average area of the neuronal cell body in P14 Tsc1-cko mouse retinas was compared with that of littermate Tsc1-het mouse retinas. Values are averages of 200 cells in 4 different mouse retinas collected from 3 independent litters. h (Left) P14 Tsc1-het and Tsc1-cko mouse retinal cells were analyzed by FACS to compare their relative cell sizes by measuring forward scatter (FSC) values. (Right) Relative sizes of Chx10:: GFP-positive bipolar cells in those littermate mouse retinas were also compared by measuring FSC values. $\mathbf{i}$ Mean sizes of the cells in the Tsc1-cko mouse retinas were obtained and shown in a graph as relative values to Tsc1-het samples $(n=3)$. Error bars in the graphs denote standard deviations $(S D)$. $P$ values are obtained by Student's $t$-test and shown in the graphs $\left({ }^{*}<0.05 ;{ }^{* \star}<0.01 ;{ }^{* \star *}<0.001\right)$ 
a

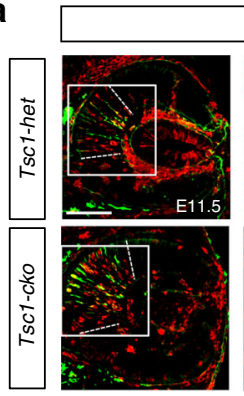

Tuj1/pS6

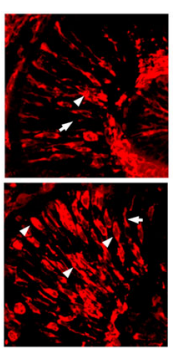

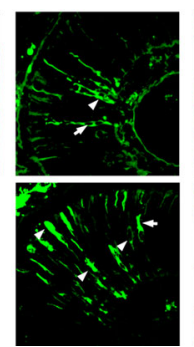

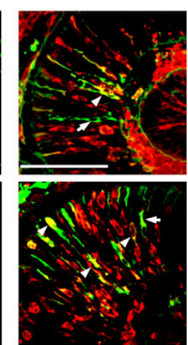

b

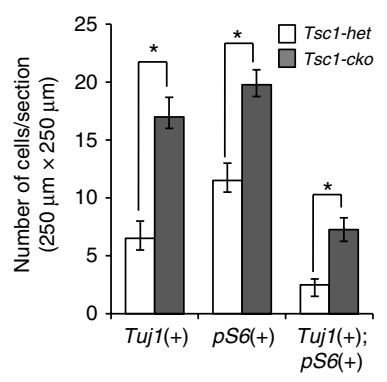

C
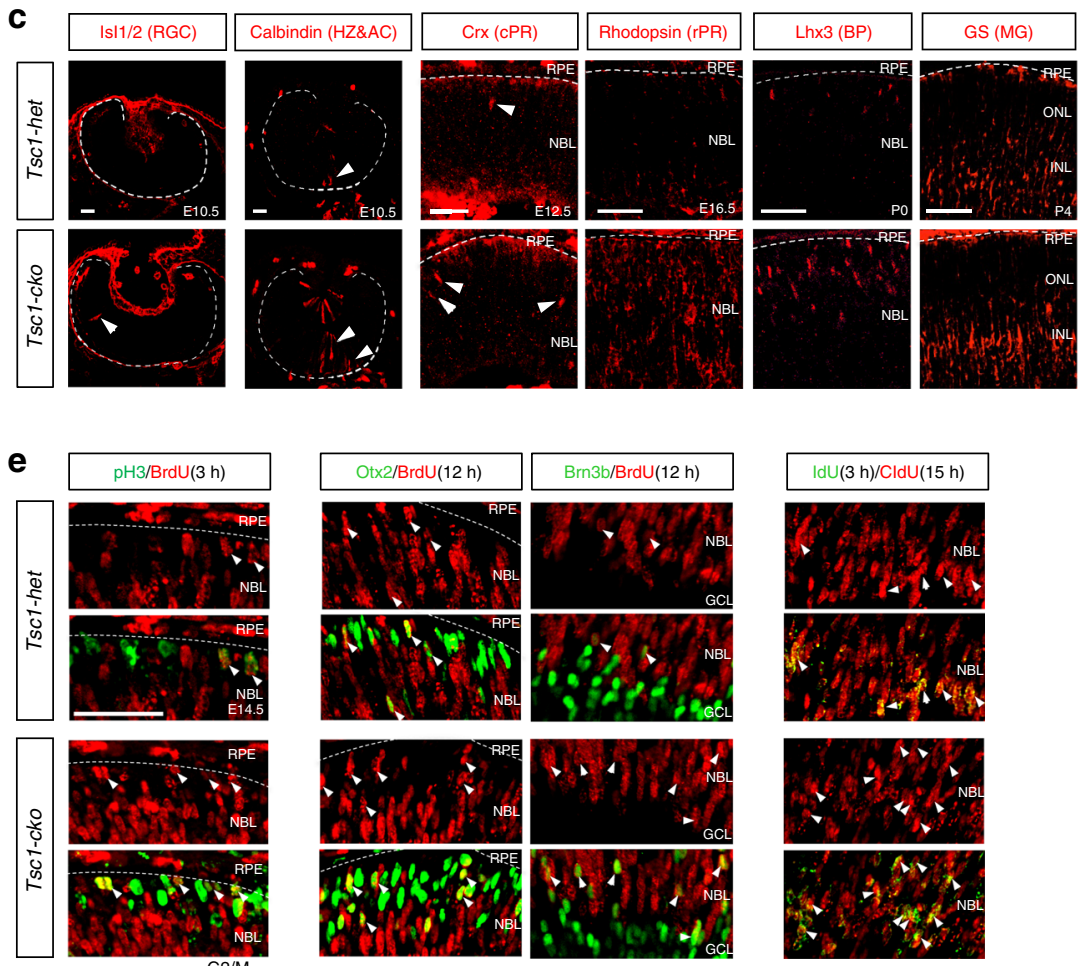

$\mathrm{S}_{1}$

$+\begin{array}{r}B^{\prime} \\ + \text { (at } 0 h \text { h } \\ +\end{array}$

(at 0 h)
+ Cldu
+

+ CldU

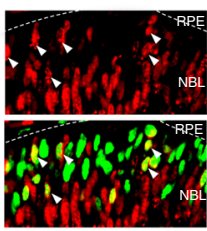

$\underset{(\mathrm{pH} 3(+))}{\mathrm{G} 2 / \mathrm{M}}$
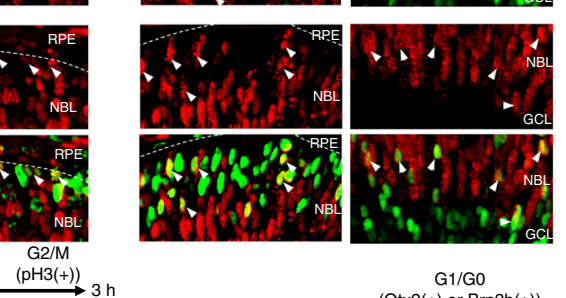

G1/G0

$(\mathrm{Ot} \times 2(+)$ or $\mathrm{Brn} 3 \mathrm{~b}(+))$
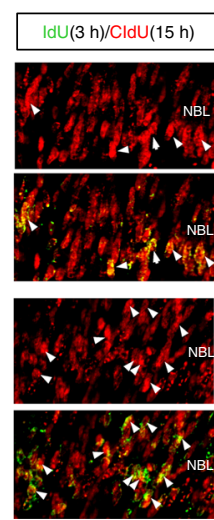

$\stackrel{(\operatorname{ldU}(+))}{\stackrel{\mathrm{S}_{2}}{\longrightarrow}}$

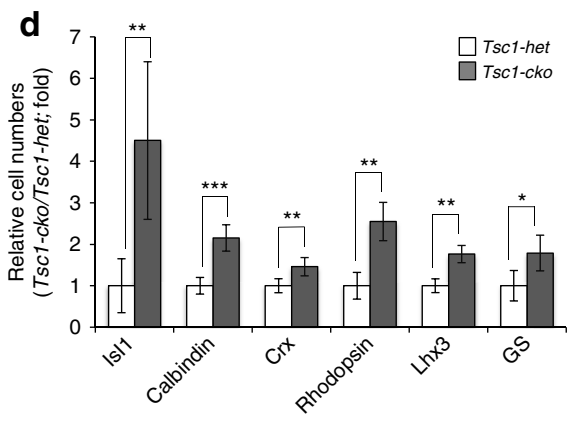

f

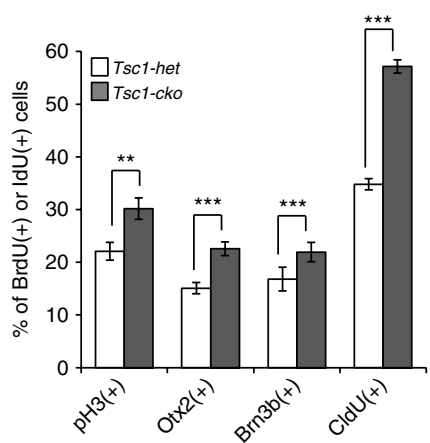

Fig. 2 mTORC1 activation accelerates RPC cell cycle and retinal development. a E11.5 Tsc1-het and Tsc1-cko littermate mouse retinal sections (coronal, 12 $\mu \mathrm{m}$ ) were co-stained with mouse anti-tubulin- $\beta I I$ antibody (Tuj1; green) and rabbit anti-pS6 antibody (red). Images in three right columns are corresponding to the lined boxes in the left. Dotted lines indicate dorsal (top) and ventral (bottom) margins of Tuj1-positive cell population. Scale bar, 100 $\mu \mathrm{m}$. b Numbers of stained retinal cells in an area $(250 \mu \mathrm{m} \times 250 \mu \mathrm{m})$ were shown in a graph. Numbers $(n)$ of retinas analyzed are 5 obtained from 3 independent litters. c Distribution of retinal neurons in littermate Tsc1-het and Tsc1-cko mouse retinas at corresponding time points, when each retinal subtype is produced prominently, was analyzed by immunostainings of various cell type-specific markers. Dotted lines mark retina-RPE borders. Arrowhead indicates marker-positive post-mitotic neurons. Scale bar, $100 \mu \mathrm{m}$. d Relative numbers of retinal cells in the Tsc1-cko mice were compared with those in Tsc1-het mice and shown in a graph. Numbers of samples analyzed are 6 (E10.5, E12.5 and E16.5) and 4 (P0 and P4) prepared from 4 and 3 independent litters, respectively. e Pregnant female mice were injected with BrdU ( $5 \mathrm{mg} / \mathrm{kg}$ ) at $3 \mathrm{~h}$ (for pH3 co-staining) or $12 \mathrm{~h}$ (for Otx2 or Brn3b costaining) prior to the collection of embryos at $14.5 \mathrm{dpc}$. Alternatively, mice were injected with $\mathrm{CldU}$ and IdU at 15 and $3 \mathrm{~h}$ prior to the collection of the embryos, respectively. Retinal sections were then co-stained with rat anti-BrdU (red) and corresponding rabbit antibodies (green) for the former analyses, and mouse anti-CldU (green) and rabbit anti-IdU (red) antibodies for the latter analysis. Arrowheads indicate cells co-expressing BrdU and corresponding markers. Scale bar, $50 \mu \mathrm{m}$. $\mathbf{f}$ Ratio of BrdU-co-expressing cells to total marker-expressing cells was obtained from 5 different samples from 3 independent litters and shown in a graph. Values in the graph are average and error bars are SD. P-values are obtained by Student's $t$-test and shown in the graphs $\left({ }^{\star}<0.05 ;{ }^{* \star}<0.01 ;{ }^{* \star \star}<0.001\right)$

generated Tsc flox/flox;Chx10-Cre (Tsc1-cko) mice lacking Tsc1/ Hamartin, which cooperates with Tsc2/Tuberin to inhibit Rheb1-dependent activation of mTORC1 specifically in the retina $^{18,19,23,24}$. Consequently, mTORC1 activity, measured indirectly by detecting the phosphorylation of ribosomal S6 protein at S235/S236 (pS6) by an mTORC1 target S6 kinase, was greatly elevated in the Tsc1-cko mouse retina in comparison to Tsc $1^{f l o x /+} ;$ Chx 10 -Cre (Tsc1-het) littermate mouse retina (Fig. 1a, b; there was no phenotypic difference among Chx10-Cre, Tsc flox) flox (Tsc1-flox), and Tsc1-het mice [data not shown]). Overall size of the eye of Tsc1-cko mice was not different significantly from Tsc1-het littermates, although the retinas of Tsc1-cko mice were 
a

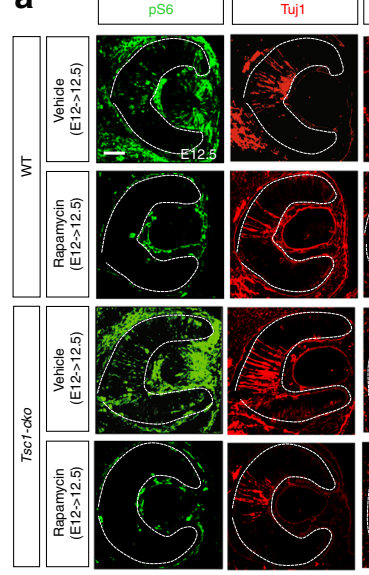

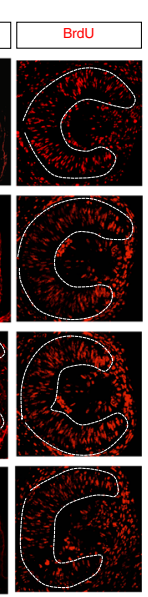

b

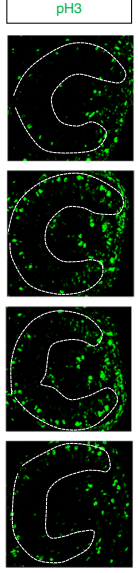

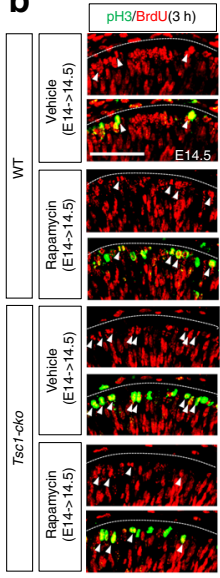

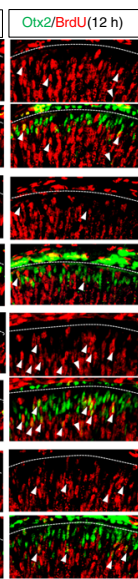

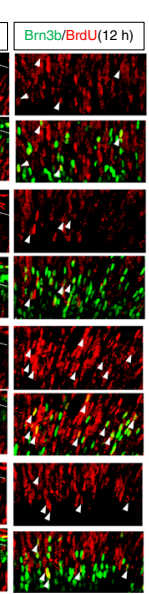

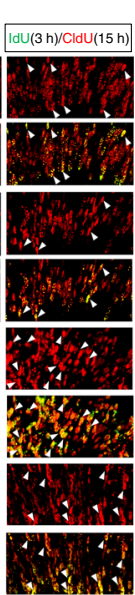

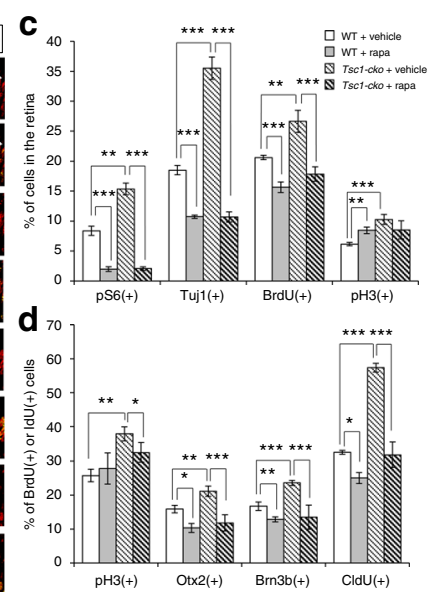

Fig. 3 mTORC1 inhibition by rapamycin inhibits RPC cell cycle and retinal development. a Pregnant mice were injected with vehicle (see Methods) or rapamycin ( $5 \mathrm{mg} / \mathrm{kg}$ ) into the peritoneum at $12 \mathrm{dpc}$, and the embryos were collected at $12.5 \mathrm{dpc}$ (E12.5). BrdU were injected at $3 \mathrm{~h}$ prior to the embryo collection. Distribution of each marker in the embryonic retinas was investigated by immunostaining with corresponding antibodies. Dotted lines mark retinal margins. Scale bar, $100 \mu \mathrm{m}$. b Cell cycle progression of embryonic RPCs in the rapamycin-injected mice was examined as described in Fig. $2 f$. Scale bar, $100 \mu \mathrm{m}$. c Percentages of cells expressing the markers in a are shown in a graph. Values are averages ( $n=5$ from 3 independent litters) and error bars denote SD. d Ratios of BrdU-co-expressing cells to total marker-expressing cells are shown in a graph ( $n=5$ from 3 independent litters). Error bars denote SD. $P$-values are obtained by one-way ANOVA test and shown in the graphs $\left({ }^{\star}<0.05 ;{ }^{\star \star}<0.01 ;{ }^{\star \star \star}<0.001\right)$

thicker than Tsc1-het littermate mouse retinas about 1.3 -fold (Fig. 1c). Cell composition of post-natal day 14 (P14) Tsc1-cko mature mouse retina was not significantly different from that of Tsc1-het littermate retina, except for RGCs that are less in Tsc1cko (Fig. 1d, e). However, mean size of cells in P14 Tsc1-cko mouse retina are over 1.2-fold larger than that in Tsc1-het littermate retina (Fig. 1f-i), suggesting that Tsc1 is important for regulating the size and morphology of retinal neurons but not their cell fates.

Using Tsc1-cko mice, we examined whether the loss of Tsc1 recapitulates developmental changes, including hyperproliferation, accelerated neurogenesis, and enhanced cell survival, observed in the Pten-cko mouse retina ${ }^{14}$. First, we investigated neurogenesis in the Tsc1-cko mouse retina by immunostaining for neuron-specific tubulin- $\beta$ III using the Tuj1 antibody. The number of Tuj1-positive retinal neurons was greatly increased in embryonic day 11.5 (E11.5) Tsc1-cko mouse retinas, expanding the neurogenic wavefront farther to the distal retina than was observed in Tsc1-het littermate mouse retinas (Fig. 2a). The larger numbers of Tuj1-positive cells showed stronger pS6 signals in Tsc1-cko mouse retinas than was observed in Tsc1-het mouse retinas (Fig. 2b), suggesting that cell autonomous activation of mTORC1 might accelerate retinal neurogenesis. Consistent with this, the numbers of islet-1-positive RGCs and calbindin-positive horizontal and amacrine cells in E10.5 Tsc1-cko mouse retinas and Crx-positive cone photoreceptors (cPRS) in E12.5 Tsc1-cko mouse retinas were increased, respectively, compared with their Tsc1-het littermate mouse retinas (Fig. 2c [left three columns], d). Not only was the production of these early-born retinal cell types more prominent in Tsc1-cko mouse retinas, so too was the production of late-born retinal cell types, including rhodopsinpositive rod photoreceptors, Lhx3-positive cone bipolar cells (BPS), and glutamine synthase-positive MG (Fig. 2c [right three columns], d). This completion of retinal development ahead of schedule is consistent with the results obtained in the Pten-cko mouse retina ${ }^{14}$.

Accelerated cell cycle progression in Tsc1-deficient RPCs. We next examined whether RPCs in the Tsc1-cko mouse retina, like those in the precociously maturating Pten-cko mouse retina ${ }^{14}$, are also hyperproliferative. Both bromodeoxyuridine (BrdU)-labeled proliferating cell number and $\mathrm{pH} 3$-positive mitotic cell number were increased in Tsc1-cko mouse retinas compared with those in Tsc1-het littermate mouse retinas during embryogenesis (Supplementary Fig. 2a-d). However, RPC population, which can be marked by Hes1 and Vsx2, is not greatly different between the Tsc1-het and Tsc1-cko littermate mice (Supplementary Fig. 2e, f). Thus, the increased numbers of proliferating cells in those mouse retinas were not resulted from the expansion RPC population, but were an indication of the increase of RPC population in active cell cycle.

The BrdU-positive cells in the ROSA26lacZ reporter (R26R), which is expressed only after Cre-mediated excision of loxPSTOP-loxP gene cassette knocked in ROSA26 gene locus ${ }^{25}$ and represents Tsc1-deficient cells in Tsc1-cko;R26lacZ/+ mouse retina, were enriched to a greater extent than those cell population in littermate Tsc1-het;R26 lacZ/+ mouse retina (Supplementary Fig. $3 c, d)$. The results suggest an autonomous hyperproliferation of R26R-positive, Tsc1-deficient RPCs resulted in faster expansion of Tsc1-deficient cell population than neighboring R26R-negative, Tsc1-positive WT RPCs (Supplementary Fig. 3a,b). This hypothesis was further supported by the results observed in P7 Tsc1 ${ }^{f / f}$; TRP1-Cre;R26 $6^{E Y F P+}$ mouse retinas, in which RPCs affected by the tyrosinase-related protein 1 (TRP1)-Cre are derived from ciliary margin and generate retinal neurons in isolated clones ${ }^{26}$. Not only total numbers of R26EYFP-positive, Cre-affected cells but also the numbers of cells comprising an individual R26EYFPpositive cell clone were elevated in P7 Tsc1 f/f;TRP1-Cre;R26 ${ }^{E Y F P /+}$ mouse retina in comparison to those in Tsc1 ${ }^{f /+}$;TRP1-Cre; $R 26^{E Y F P /+}$ littermate mouse retina (Supplementary Fig. $3 \mathrm{e}-\mathrm{h}$ ). Collectively, these results therefore account for the fact that the R26R-positive, Tsc1-deficient population occupies $~ 90 \%$ of the retinal area of P14 Tsc1-cko;R26 ${ }^{\text {lacZ/+ }}$ mice, whereas the R26Rpositive, Tsc1-heterzygous population comprises $\sim 75 \%$ in $T s c 1$ het; $R 26^{\text {lacZ/+ }}$ littermate mouse retina (Supplementary Fig. 3a, b).

We next assessed the speed of RPC division in mouse retinas indirectly by counting the number of RPCs that completed first round of cell cycle after incorporating chlorodeoxy-uridine $(\mathrm{CldU})$ into their chromosomes for $12 \mathrm{~h}$ and then proceeded to 
a

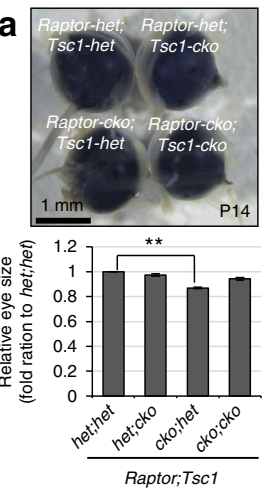

e

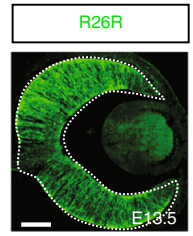

वृ.

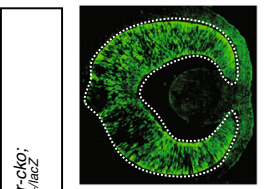

这这 b
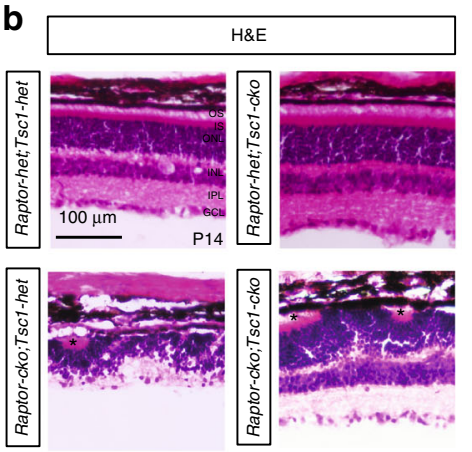

c
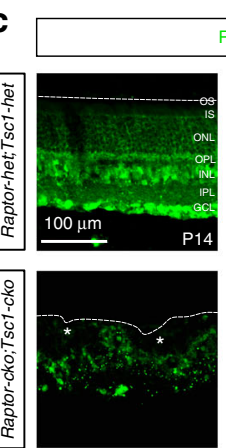
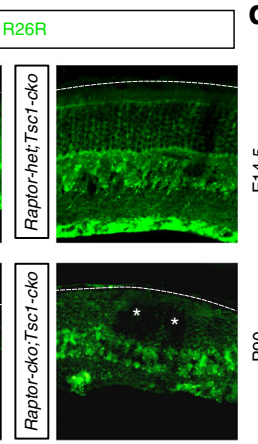

d
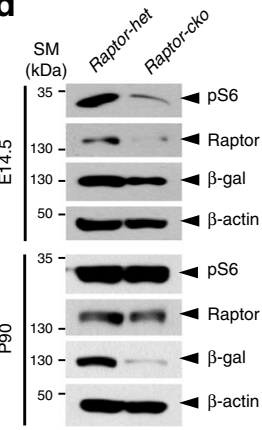
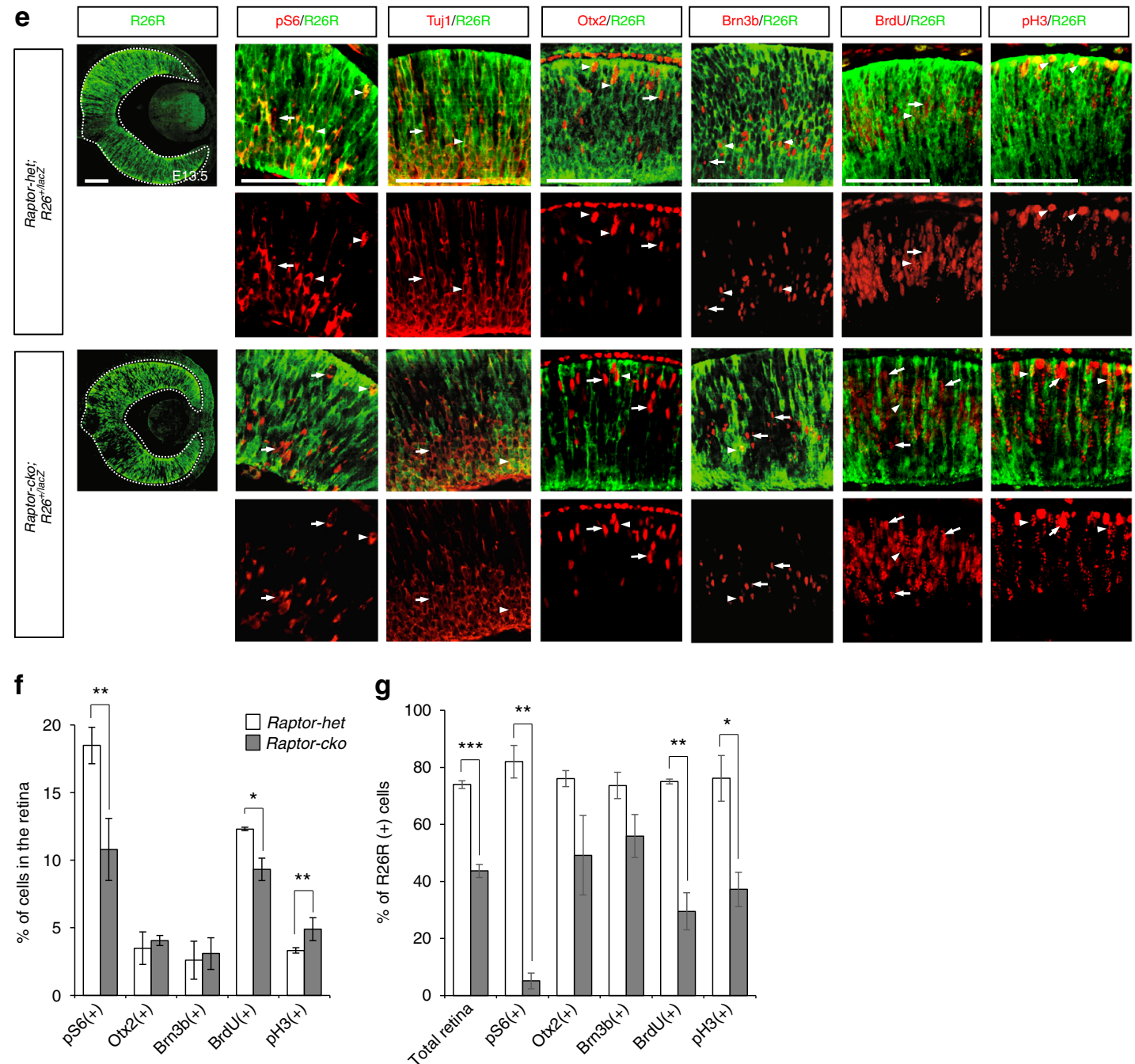

Fig. 4 Raptor-deficient RPC fails to progress cell cycle. a Top, images of P30 Raptor-het;Tsc1-dhet, Raptor-het;Tsc1-cko, Raptor-cko;Tsc1-het, and Raptor-cko; Tsc1-cko littermate mouse eyes. Bottom, relative eye sizes are shown in a graph by comparing average diameter of the eyes. Number of eyes measured the

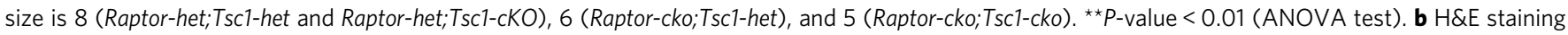
images of P14 Raptor-het;Tsc1-het, Raptor-het;Tsc1-cko, Raptor-cko;Tsc1-het, and Raptor-cko;Tsc1-cko littermate mouse retinal sections. c Distribution of Raptordeficient cells in P14 littermate mouse retina was visualized indirectly by immunodetection of R26R, which is expressed from Cre-recombined R26lacZ loci.

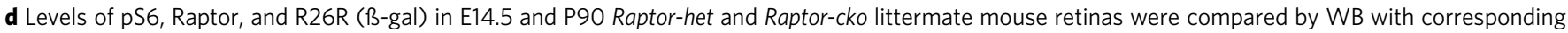
antibodies. Relative amounts of protein loaded in each lane were determined by WB detection of $B$-actin. e Distributions of retinal markers in E13.5 Raptorhet; $R 26 R^{+/ l a c Z}$ and Raptor-cko; $R 26 R^{+/ l a c Z}$ littermate mouse embryonic retinas were examined by immunostaining with corresponding antibodies. The R26Rpositive cells (green) are potentially the Raptor-deficient cells. BrdU was injected to pregnant mice at $3 \mathrm{~h}$ prior to embryo collection. Arrows and arrowheads indicate R26R-negative and R26R-positive cells that express specific markers (red), respectively. Scale bar, 100 mm. f Percentage of retinal cells expressing each marker in the retinas was provided in a graph (representative images are shown in Supplementary Fig. 7). $\mathbf{g}$ Ratios of R26R co-expressing cells to total marker-expressing cells in a are shown in a graph. Numbers of samples used for obtaining graphs in $\mathbf{b}$ and $\mathbf{c}$ are 4 (from 3 independent litters); error bars denote SD. P-values are obtained by Student's t-test and shown in the graphs $\left({ }^{\star}<0.05 ;{ }^{\star \star}<0.01 ;{ }^{\star \star \star}<0.001\right)$ 
second round to incorporating iododeoxyuridine (IdU) for $3 \mathrm{~h}$. The ratio of CldU/IdU double-labeled cells, which are the population had entered into second round of cell cycle after completing first round, to total CldU-labeled proliferating cells was higher in E14.5 Tsc1-cko mouse retinas than in Tsc1-het littermate mouse retinas (Fig. 2e [rightmost column], f). To dissect the cell cycle of mouse RPCs, we also counted the number of pH3-positive mitotic cells that had entered G2 interphase and/ or mitotic (M) phases of the cell cycle within $3 \mathrm{~h}$ after incorporating BrdU during $\mathrm{S}$ phase. The number of $\mathrm{pH} 3$ positive cells co-labeled with BrdU was higher in E14.5 Tsc1-cko mouse retinas than in Tsc1-het littermate mouse retinas (Fig. 2e [leftmost column], f). The population of Brn3b-expressing RGCs and Otx2-positive cPRs that incorporated BrdU to their chromosomes during a $12-\mathrm{h}$ period prior to exiting the cell cycle were also increased in Tsc1-cko mouse retinas (Fig. 2e [two center columns], f). These results suggest that daughter cells of the Tsc1deficient RPCs not only progress to next round of cell cycle faster than Tsc1-het cells, they also exit from the cell cycle to produce more neurons during the same time period. However, overproduced retinal cells from Tsc1-cko mouse RPCs were eliminated by apoptotic cell death (Supplementary Fig. 4), adjusting the final number of retinal cells to a value similar to that in Tsc1-het mouse retinas (Fig. 1d, e).

Rapamycin inhibits retinal neurogenesis by interfering with progenitor cell cycle progression. We next tested whether inhibition of mTORC1 exerted effects on RPC proliferation and retinal neurogenesis opposite those of mTORC1 activation. To accomplish this, we injected pregnant WT and Tsc1-cko female mice with rapamycin, a chemical inhibitor of mTORC1 $1^{27}$ 28, at E12 days post coitum. By E12.5, pS6 signals had disappeared in embryonic retinas isolated from the rapamycin-injected mice regardless of their genotypes (Fig. 3a [leftmost column], f), indicating successful inhibition of mTORC1 by rapamycin. Rapamycin injection also significantly decreased the number of Tuj1-positive retinal neurons in those mouse embryos compared with that observed in retinas isolated from vehicle-injected WT and Tsc1-cko mouse embryos at the same developmental stage (Fig. 3a [second left column], c). Similar neurogenic defects were also observed in mouse embryos treated with an mTOR inhibitor, Torin1 (Supplementary Fig. 5a,b).

Inhibition of mTORC1 not only influenced retinal neurogenesis but it also affected RPC proliferation, decreasing the number of BrdU-labeled cells in WT and Tsc1-cko mouse embryonic retinas (Fig. 3a [second right column], c). Interestingly, the number of $\mathrm{pH} 3$-positive mitotic RPCs was rather increased in embryonic retinas from rapamycin-injected mice (Fig. 3a [rightmost column], c). The ratio of BrdU;pH3 double-positive cells to total $\mathrm{pH} 3$-positive cells, however, was not accordingly increased, but was decreased, in the rapamycin-treated E14.5 WT mouse retinas (Fig. 3b [leftmost column], d). The rapamycin treatment also decreased the BrdU;pH3 double-positive cell population in Tsc1-cko mouse retinas compared to that in vehicle-treated E14.5 Tsc1-cko mouse retinas (Fig. 3b [bottom row of leftmost column], d). The populations of CldU;IdU double-positive RPCs, BrdU; Otx2 double-positive post-mitotic photoreceptors, and BrdU; Brn3b double-positive post-mitotic RGCs were also all decreased in rapamycin-treated groups regardless of genotype (Fig. $3 \mathrm{~b}$ [right three columns], d). These results suggest that increase of $\mathrm{pH} 3$ is a reflection of mitotic cell cycle arrest in the rapamycin-treated mouse retinas while decrease of BrdU represents reduced entry to $S$ phase from G1. Collectively, these results imply that rapamycin treatment interferes with cell cycle progression of RPCs and consequently impairs retinal neurogenesis as well as cell cycle reentry of RPC.

Cell cycle progression is defective in Raptor-deficient RPCs. The mTORC1 complex contains an essential component Raptor, which is replaced by Rictor in mTORC $2^{29,30}$. To investigate whether rapamycin-induced suppression of RPC proliferation and retinal neurogenesis were specifically mediated by inhibition of mTORC1, we examined retinal development in Raptorflox/flox; Chx10-Cre (Raptor-cko) mice, which lack Raptor and lose mTORC1 activity in RPCs and descendent retinal neurons. The average size of Raptor-cko mouse eyes was about $87 \%$ that of Raptor-het littermate eyes; their retinas were also thinner than normal and showed multiple rosette structures (Fig. $4 \mathrm{a}, \mathrm{b}$ ). Interestingly, R26R-positive cells, which are Raptor-deficient, were hardly detectable in mature Raptor-cko mouse retinas (Fig. 4c, d), but were present in significant numbers in the embryonic stages (Fig. 4d, e [leftmost column]). However, there were already significantly fewer R26R-positive cells in Raptor-cko embryonic mouse retinas than in Raptor-het littermate mouse retinas (Supplementary Fig. 6d). Not only total apoptotic cell numbers but also R26R-positive apoptotic cell population were significantly increased in the Raptor-cko embryonic mouse retinas (Supplementary Fig. 6a, b, c, e, f), implicating preferential loss of Raptor-deficient cells by cell death. The results suggest that Raptor-deficient cells are eliminated by apoptosis in the embryonic mouse retinas, resulting in the depletion of R26Rpositive Raptor-deficient cell population in the post-natal Raptorcko mouse retinas.

In contrast to the lack of pS6 signal in rapamycin-treated retinas, pS6-positive cells were still present at reduced number in E13.5 Raptor-cko mouse retinas (Fig. 4e; Supplementary Fig. 7). In addition, the numbers of neurons positive for Tuj1, Otx2, or Brn3b in the retina were not changed to as great an extent as was the case in rapamycin-treated retinas (Fig. 4e, f; Supplementary Fig. 7). However, a comparison of $\mathrm{pS} 6$ and R26R positivity in Raptor-cko mouse retinas revealed that pS6 signals were selectively enriched in R26R-negative WT retinal cells (Fig. 4e, g). A majority of cells expressing those neuronal markers in Raptor-cko mouse retinas were also negative for R26R (Fig. 4e [three center columns], g), suggesting that the loss of Raptor and consequent inactivation of mTORC1 interfered with retinal neurogenesis. The R26R-positive cells also showed a reduced ability to incorporate BrdU and exhibited diminished $\mathrm{pH} 3$ expression (Fig. 4e, g), indicating a proliferation defect in Raptor-deficient cells. In support of complementary expansion of WT cells in the retina, the numbers of mitotic RPCs ( $\mathrm{pH} 3$ positive) and retinal neurons (Brn3b-positive and Otx2-positive), which incorporated BrdU for 3 and $12 \mathrm{~h}$, respectively, were higher in the R26R-negative WT cell population than in the R26Rpositive Raptor-deficient cell population in the E13.5 Raptor-cko mouse retina (Fig. 4g). Collectively, these results suggest that inactivation of mTORC1, either by rapamycin treatment or Raptor deletion, inhibits RPC cell cycle progression for neurogenesis as well as self-renewal, whereas activation of mTORC1 promotes those.

Cyclin turnover in RPCs is sensitive to mTORC1 activity. Mitotic cell cycle progression requires accumulation of cyclin $\mathrm{B}$ $(\mathrm{CcnB})$ and consequent activation of cyclin-dependent kinase 1 (Cdk1), which promotes mitosis by phosphorylating various target proteins in $\mathrm{G} 2 / \mathrm{M}$ phases of the cell cycle ${ }^{31-33}$. In the same manner, CcnE and CcnA trigger the progression to S and G2 phases from $\mathrm{Gl}$ and $\mathrm{S}$ phases, respectively, by activating $\mathrm{Cdk} 2^{33}, 34$. Consistent with a supportive role of mTORC1 in 

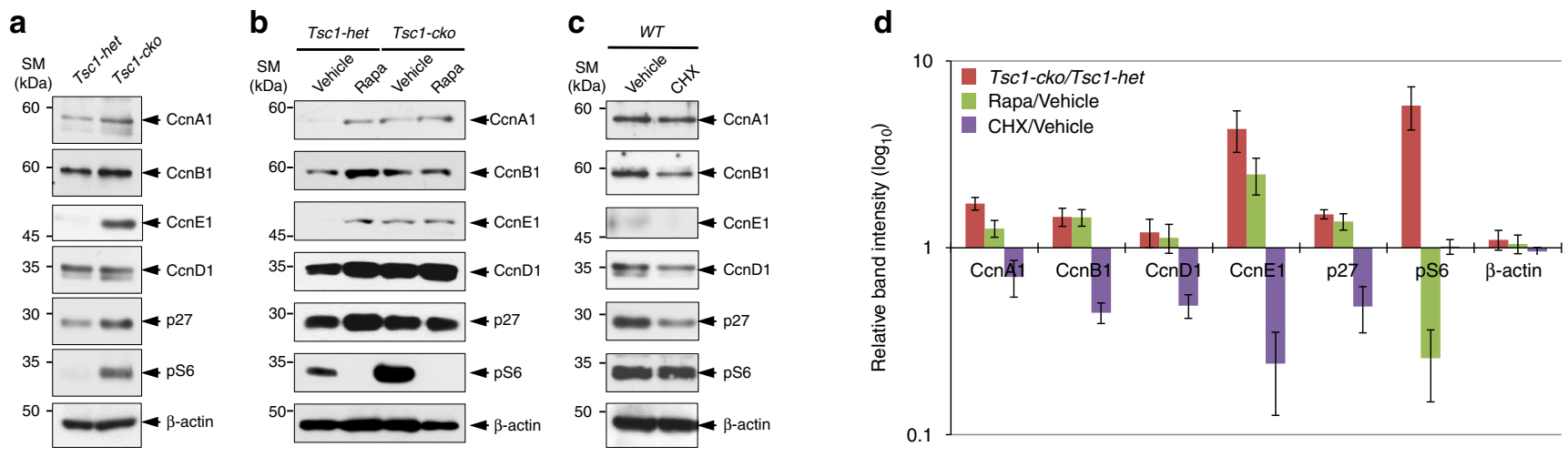

e

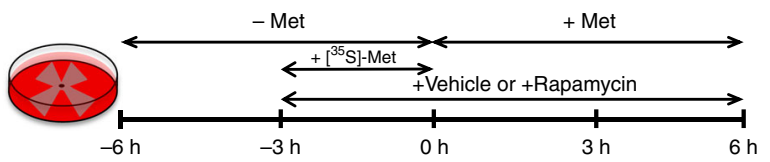

f
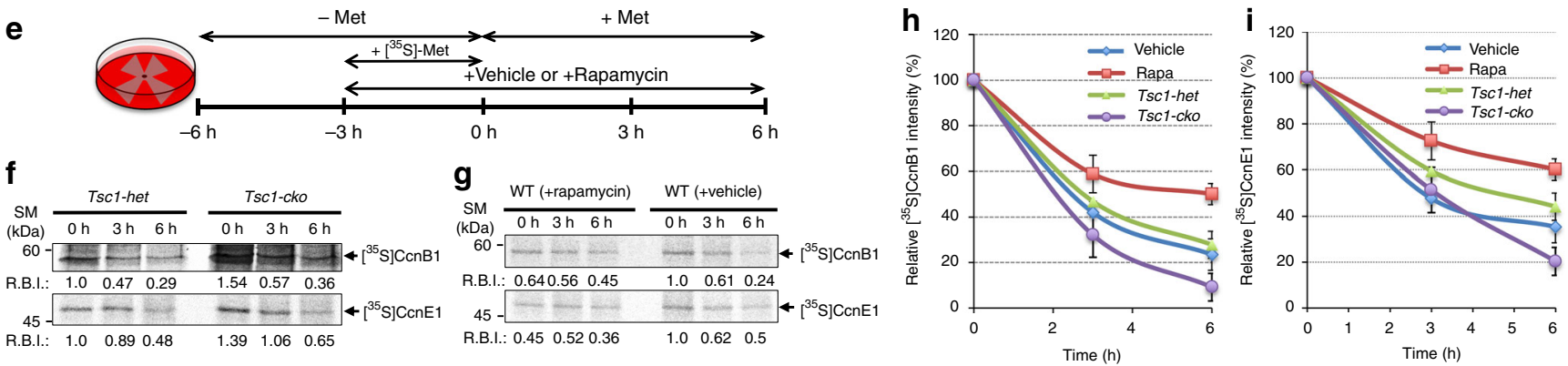

Fig. 5 Cyclin turnover in RPC is sensitive to mTORC1 activity. a Proteins (20 $\mu \mathrm{g})$ of cell lysates of E14.5 Tsc1-het and Tsc1-cko littermate mouse retinas were analyzed by WB with corresponding antibodies. Alternatively, pregnant mice can produce either Tsc1-het or Tsc1-cko mice were injected with vehicles (control in $\mathbf{b}$ and $\mathbf{c}$ ), rapamycin $(5 \mathrm{mg} / \mathrm{kg}$; b), or cycloheximide (CHX; $5 \mathrm{mg} / \mathrm{kg}$; c) at $14 \mathrm{dpc}$ prior to collecting the embryonic retina at $14.5 \mathrm{dpc}$ for WB analyses. $\mathbf{d}$ Image pixels of WB bands were calculated by the ImageJ software and relative intensities were obtained by comparing the pixel numbers with those of control samples (TSC1-het retina for Tsc1-cko; vehicle-injected retinas for rapamycin and CHX). Values are average measurements of 3 independent WB results. Error bars denote SD. e Experimental schedule for labeling and chasing of newly synthesized cyclin proteins in mouse retinal explants. E13.5 littermate retinal explants were incubated in methionine (Met)-free growth media for $3 \mathrm{~h}$ and then added with $\left.{ }^{35} \mathrm{~S}\right]-\mathrm{Met}(250 \mu \mathrm{Ci} / \mathrm{ml})$ for $3 \mathrm{~h}$ to label newly synthesized proteins in the retinas. The explants were then incubated in normal growth media containing unlabeled Met for the indicated time. For the analysis in $\mathbf{g}$, WT C57BL6/J mouse retinal explants were added with growth media containing vehicle or rapamycin as indicated. $\mathbf{f}, \mathbf{g}$ CcnB1 and CcnE1 in the retinal explants were isolated by immunoprecipitation with corresponding antibodies and the immunoprecipitates were analyzed by $10 \%$ SDS-PAGE followed by detection of radioactivities of ${ }^{35} \mathrm{~S}$ ]-labeled protein bands using BAS-7000 (Fuji Inc.). Relative band intensities (RBI) of [ $\left.{ }^{35} \mathrm{~S}\right]-\mathrm{C} \mathrm{cnB} 1$ and $\left[{ }^{35} \mathrm{~S}\right]$ $\mathrm{CcnB1}$ were obtained by comparing the image pixel numbers $\left[{ }^{35} \mathrm{~S}\right]-\mathrm{CcnB} 1$ and $\left[{ }^{35} \mathrm{~S}\right]-\mathrm{CcnB} 1$ bands of each sample with $T s c 1$-het (f) and vehicle-treated $(\mathbf{g})$ samples at time $\mathrm{O}\left(\mathrm{O}\right.$ h) using Image J software, and presented below the band images. To examine the decay rates of $\left[{ }^{35} \mathrm{~S}\right]-\mathrm{labeled} \mathrm{CcnB1}(\mathbf{h})$ and $\mathrm{CcnE1}(\mathbf{i})$ in Tsc1-cko and rapamycin-treated mouse retinas, relative intensities against the values at $\mathrm{O} h$ were obtained and shown in the graphs. The values are average band intensity obtained from 4 independent experiments. Error bars denote SD

mitotic cell cycle progression ${ }^{27,35}$, the level of CcnB1 in E14.5 Tsc1-cko mouse retinas was higher than that in Tsc1-het littermate mouse retinas (Fig. 5a, d; Supplementary Fig. 8). The levels of CcnE1 and CcnA1 were also commonly increased in the E14.5 Tsc1-cko mouse retinas, implicating enhanced G1-to-S and S-toG2 cell cycle progression of mTORC1-hyperactive Tsc1-deficient RPCs. However, the levels of CcnD1, which is expressed in $\mathrm{RPCs}^{36}$, were not significantly different between E14.5 Tsc1-het and Tsc1-cko mouse retinas (Fig. 5a, d), in consistent with indifferent RPC population in those littermate mouse retinas (Supplementary Fig. 2e, f).

Unexpectedly, rapamycin treatment, which interfered with cell cycle progression in mouse embryonic RPCs (Fig. 3b, d), did not decrease cyclin levels in E14.5 mouse retinas regardless of genotypes (Fig. 5b, d). It rather increased the levels of cyclin proteins in mouse retinas (Fig. 5b, d), without correspondingly increasing cyclin mRNA levels (Supplementary Fig. 8). Given the negative effects of rapamycin on translation ${ }^{37}$, the results were quite surprising. We thus hypothesized that rapamycin treatment might accumulate cyclin proteins by interfering with the synthesis of the proteins that inhibit cyclin synthesis or promote cyclin decay. However, in opposite to the effects of rapamycin, the levels of cyclin proteins were commonly decreased in embryonic retinas isolated from mice injected with a general translation inhibitor cycloheximide (CHX; Fig. 5c, d). These results therefore imply that cyclin accumulation in rapamycin-treated mouse retina was not resulted from the inhibitory effects of rapamycin on translation.

Cell cycle progression is not only triggered by the synthesis of cell cycle phase-specific cyclins, it is also coupled to the degradation of cyclins that had acted in previous cell cycle phases $^{34}$. Accordingly, mTORC1 might promote RPC cycle progression by regulating cyclin protein degradation as well as synthesis. Hence, mTORC1 inactivation by rapamycin possibly resulted in cyclin accumulation through inhibition of degradation of the proteins. To test this hypothesis, we examined the rates of cyclin protein degradation in mouse embryonic retinal explants by pulse labeling newly synthesized cellular proteins with $\left[{ }^{35} \mathrm{~S}\right]$ methionine and subsequently chasing with unlabeled methionine (Fig. 5e). The relative amounts of $\left.{ }^{35} \mathrm{~S}\right]$-labeled $\mathrm{CcnB} 1$ and CcnE1 synthesized over a 3-h labeling period were higher in Tsc1cko retinal explants than in Tsc1-het littermate retinal explants (Fig. 5f, see relative band intensity (RBI) values), reaffirming the translation-stimulating activity of $\mathrm{mTORC} 1^{37}$. However, the 

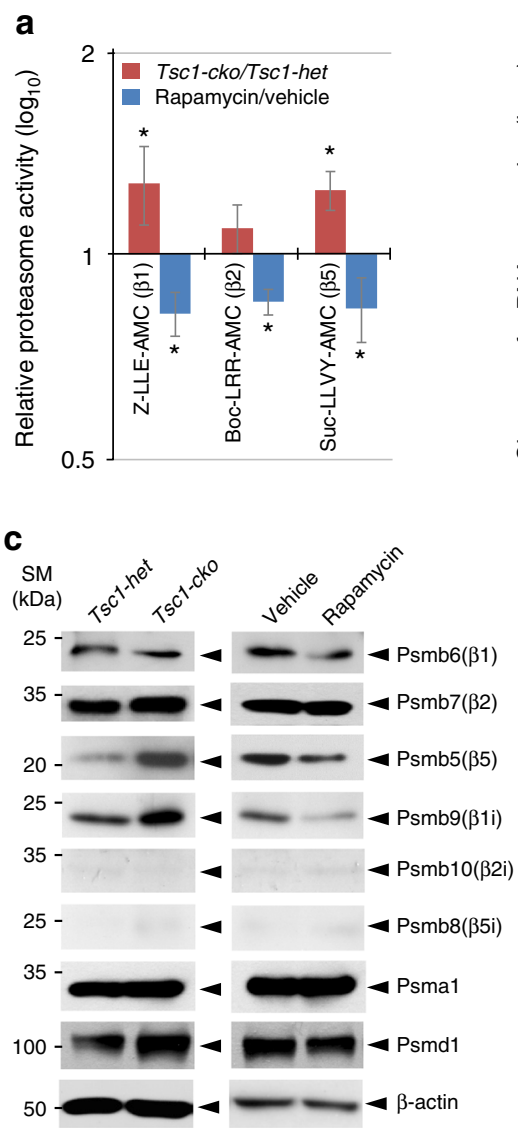

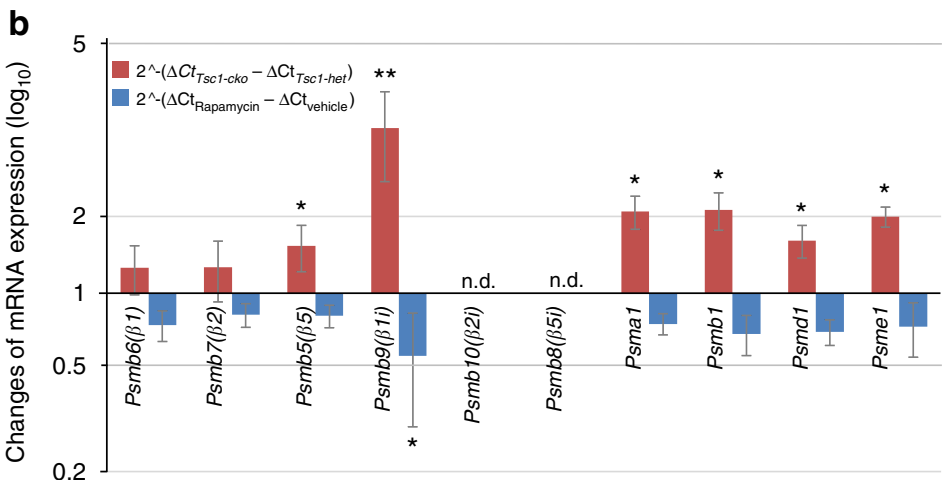

d

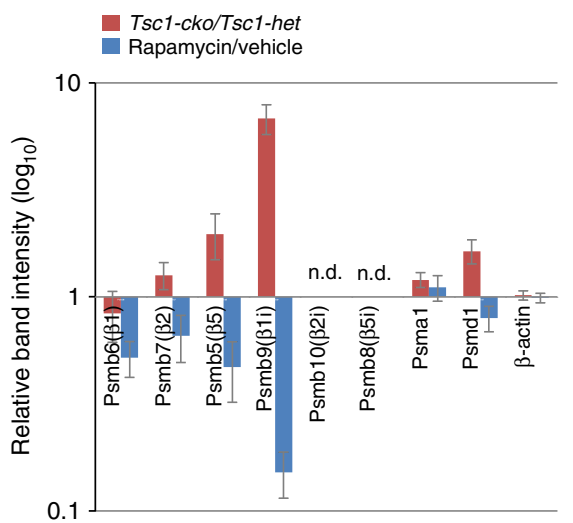

e

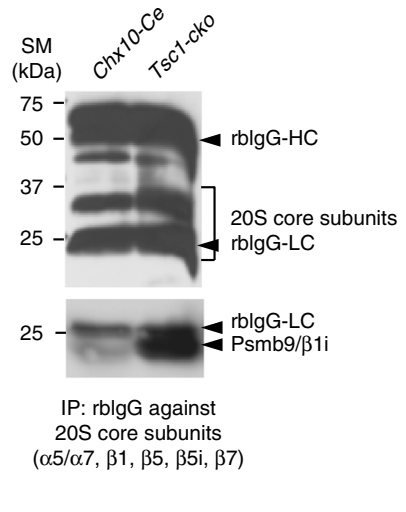

Fig. 6 mTORC1-induced developmental acceleration of the retina requires an immunoproteasome subunit Psmb9. a The 20S proteasomes were purified from E14.5 Tsc1-het and Tsc1-cko littermate mouse retinas by 20S Proteasome Purification Kit ${ }^{\oplus}$ (Enzo Life Sciences), and caspase-like ( $\left.\beta 1 / \beta 1 i\right)$, trypsin-like $(\beta 2 / \beta 2 \mathrm{i})$, and chymotrypsin-like $(\beta 5 / \beta 5 \mathrm{i})$ activities of the purified proteasomes were analyzed by measuring fluorescent intensities produced by cleaved peptide substrates Z-LLE-AMC ( $\beta 1 / \beta 1 i)$, Bz-VGF-AMC $(\beta 2 / \beta 2 \mathrm{i})$, and Scu-LLVY-AMC $(\beta 5 / \beta 5 \mathrm{i})$ (see details in Methods). The values are averages and error bars denote SD ( $n=3 ; 3$ independent litters). b Relative mRNA levels of 20 S proteasome subunits in E14.5 Tsc1-cko and rapamycin-treated mouse retinas were obtained by comparing real-time quantitative PCR (RT-qPCR) values with those of Tsc1-het and vehicle-treated mouse retinas at the same age. The values are averages obtained by 6 (Tsc1-cko/Tsc1-het) and 7 (rapamycin/vehicle) independent measurements with mRNA isolated from 4 (Tsc1-cko/Tsc1het) and 6 (rapamycin/vehicle) independent batches. c Relative levels of 205 proteasome subunit proteins in the mouse retinas were also examined by WB with corresponding antibodies. d Image pixels of WB bands in Tsc1-cko samples were calculated by the ImageJ software and relative intensities were obtained by comparing the pixel numbers with those Tsc1-het samples. Values are average measurements of 4 independent WB results. e Incorporation of Psmb9 into the proteasome was examined by WB detection of Psmb9 in 205 proteasome core complex, which was isolated by the 205 Proteasome Purification Kit. $P$-values are obtained by Student's $t$-test and shown in the graphs $\left({ }^{\star}<0.05 ;{ }^{\star \star}<0.01\right)$

levels after a 3-h chase period were not greatly different between the two genotypes, and were further decreased in Tsc1-cko retinal explants after a 6-h chase (Fig. 5h, i). These results suggest that $\left[{ }^{35} \mathrm{~S}\right]$-labeled cyclins persist for a shorter period of time in Tsc1cko mouse retinas than in Tsc1-het littermate mouse retinas.

Conversely, the relative amounts of $\left.{ }^{35} \mathrm{~S}\right]$-labeled cyclins in retinal explants treated with rapamycin during a 3-h labeling period were lower than those in vehicle-treated retinal explants (Fig. $5 \mathrm{~g}$, see RBI values). The decay of $\left.{ }^{35} \mathrm{~S}\right]$-labeled cyclins was also slower in the rapamycin-treated retinal explants. About 50\% of the $\left[{ }^{35} \mathrm{~S}\right]$-labeled CcnB1 was still detectable in rapamycintreated retinas $6 \mathrm{~h}$ post incubation, whereas only $23 \%$ of $\left[{ }^{35} \mathrm{~S}\right]-$ labeled CcnB1 remained in vehicle-treated samples at the same time point (Fig. 5h). A similar decay pattern was also observed from $\left[{ }^{35} \mathrm{~S}\right]$-labeled $\mathrm{C} c \mathrm{nE} 1$, suggesting that the turnover rate of CcnE1 is also sensitive to mTORC1 activity (Fig. 5i). However, the total amount of $\left[{ }^{35} \mathrm{~S}\right]$-labeled protein in the retinas was not sensitive to mTORC1 activity (Supplementary Fig. 9a, b), suggesting that mTORC1-regulated protein decay is not applicable to all proteins, but might be specific for certain proteins, including $\mathrm{CcnB1}$ and $\mathrm{C} \mathrm{cnE} 1$. Taken together, these results suggest that mTORC1 not only facilitates the synthesis of cyclins, it also promotes the degradation of these proteins. The short oscillatory cycle of cyclin synthesis and degradation might drive mTORC1activated RPCs to progress through the cell cycle faster than control RPCs.

Elevated proteasomal activity in the Tsc1-cko mouse retina. The decay of cyclins during the cell cycle is mainly mediated by ubiquitin-dependent proteasomal degradation ${ }^{34}$, suggesting that enhanced cyclin turnover in the Tsc1-cko mouse retina might result from enhanced ubiquitination of cyclins. The relative level of ubiquitinated CcnB1 was, however, lower in E14.5 Tsc1-cko mouse retinas than in littermate Tsc1-het mouse retinas-the inverse of the higher CcnB1 level in Tsc1-cko mouse retinas (Supplementary Fig. 10a, b). On the contrary, the levels of other ubiquitinated proteins were not significantly different between the Tsc1-het and Tsc1-cko littermate mouse retinas (Supplementary Fig. 10f). Ubiquitinated CcnB1 levels became 

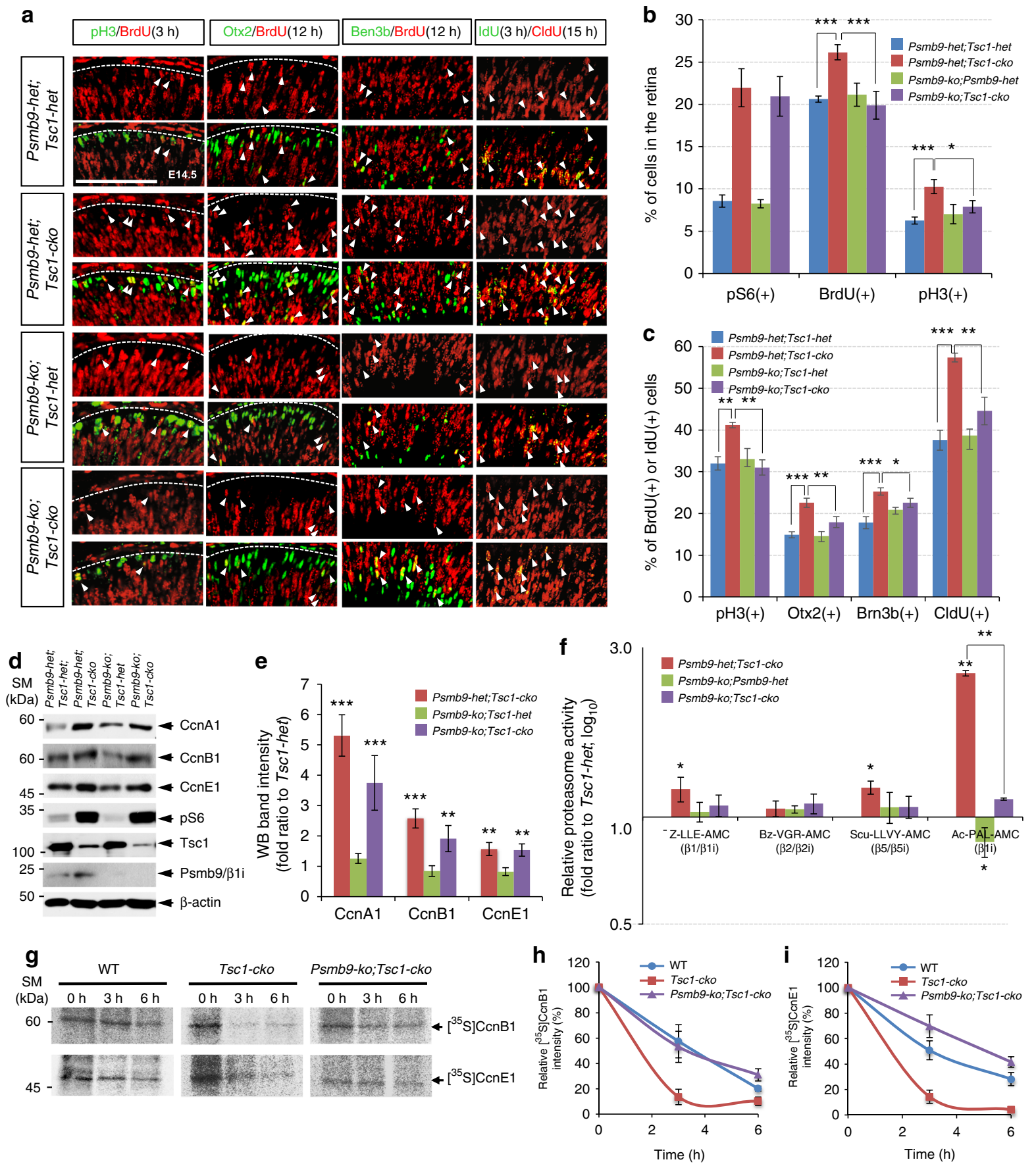

Fig. 7 Psmb9 is necessary for the accelerated cyclin turnover in Tsc1-cko RPC. a Cell cycle progression of RPCs in E14.5 mouse retinas with combinatorial Tsc1-cko and Psmb9-ko alleles was examined as described in Fig. 2f. Scale bar, $100 \mu \mathrm{m}$. b Average percentage of cells expressing each marker in the retinas was provided in a graph (representative images are shown in Supplementary Fig. 12a). c Ratio of BrdU-co-expressing cells to total marker-expressing cells in (a) was also shown in a graph. Numbers of mice used for $\mathbf{b}$ and $\mathbf{c}$ are 4 (Tsc1-cko;Psmb9-ko) or 5 (the rest genotypes) and from 4 independent litters; error bars denote SD. $\mathbf{d}$ Retinal cell lysates ( $20 \mu \mathrm{g}$ proteins/lane) obtained from E14.5 littermate mouse embryos were analyzed by WB with corresponding antibodies. e Image pixels of WB bands in each retinal sample were calculated by ImageJ software and relative intensities were obtained by comparing the pixel numbers with those of Psmb9-het;Tsc1-het samples. Values are average measurements of 3 independent WB results. Error bars denote SD. $\mathbf{f}$ Activities of the purified proteasomes were analyzed as described in Fig. 6a. The values are averages and error bars denote SD ( $n=4 ; 2$ independent litters). $\mathbf{g}$ Synthesis and degradation of $\mathrm{CcnB1}$ in the mouse retinas were analyzed by $\left.{ }^{35} \mathrm{~S}\right]-$ Met pulse labeling and chasing experiments explained in Fig. 5 e. Relative radioactivities of $\left[{ }^{35} \mathrm{~S}\right]$-labeled $\mathrm{C} \mathrm{cnB} 1(\mathbf{h})$ and $\mathrm{CcnE} 1$ (i) bands in the retinal explants were obtained by comparing corresponding bands at $\mathrm{O}$. The values are average obtained from 3 independent experiments. Error bars denote SD. P-values are obtained by one-way ANOVA test and shown in the graphs $\left({ }^{\star}<0.05 ;{ }^{* \star}<0.01 ;{ }^{* \star *}<0.001\right)$

indistinguishable between E14.5 Tsc1-het and Tsc1-cko littermate mouse retinas after the inhibition of the proteasomes by MG-132. The results implicate that the amounts of ubiquitinated $\mathrm{CcnB1}$ gained after MG-132 treatment were higher in Tsc1-cko mouse retinas in comparison to those in Tsc1-het littermates (Supplementary Fig. 10a, b, e). Together, the results suggest that degradation of ubiquitinated CcnB1 is more efficient in the Tsc1-cko mouse retinas than that in the Tsc1-het mouse retinas. 
Conversely, the levels of ubiquitinated CcnB1 in mouse embryonic retinas were increased by rapamycin treatment (Supplementary Fig. 10c). MG-132 treatment did not cause further accumulation of ubiquitinated proteins in rapamycininjected mouse retinas, suggesting that proteasomal degradation of ubiquitinated CcnB1 was already inhibited in the rapamycintreated retinas (Supplementary Fig. 10c-e). Collectively, these results suggest that proteasome-mediated degradation of ubiquitinated $\mathrm{CcnB1}$, but not the rate of $\mathrm{CcnB1}$ ubiquitination, is sensitive to mTORC1 activity.

In support of this, the activities of caspase-like (responsible by $\beta 1$ subunit) and chymotrypsin-like proteases (responsible by $\beta 5$ subunit), which constitute the catalytic subunits of the $20 \mathrm{~S}$ proteasome core particle together with trypsin-like (responsible by $\beta 2$ subunit) proteases ${ }^{38}$, were $\sim 1.2$-fold higher in E14.5 Tsc1cko mouse retinas than in Tsc1-het littermate mouse retinas (Fig. 6a). Conversely, rapamycin treatment decreased the proteasome activities in E14.5 mouse retinas to about $80 \%$ of that observed in vehicle-treated retinas (Fig. 6a). Taken together with the $\left[{ }^{35} \mathrm{~S}\right]$-Met pulse-chase results (Fig. 5f), these findings suggest that elevated proteasome activity might be responsible for the accelerated degradation of CcnB1 in the Tsc1-cko mouse retina.

mTORC1 stimulates proteasome gene expression in the mouse retina. It was reported that expression of $20 \mathrm{~S}$ proteasome core particle subunit genes is enhanced in Tsc2-ko mouse embryonic fibroblasts and adult mouse brain neurons, in which mTORC1 activity is also increased ${ }^{39}$. Thus, to determine whether this mTORC1-induced proteasome gene expression might account for the increased proteasome activity in Tsc1-cko retinas, we examined the levels of proteasome subunit mRNAs and proteins in E14.5 Tsc1-het and Tsc1-cko mouse retinas. The relative mRNA levels of 20S constitutive proteasome catalytic subunits $\beta 1$ (Psmb6), $\beta 2$ (Psmb7), and $\beta 5$ (Psmb5) were changed insignificantly or increased $<1.5$-fold in E14.5 Tsc1-cko mouse retinas compared with Tsc1-het littermate mouse retinas (Fig. 6b). The mRNA levels of these proteasome subunits were decreased mildly (to $\sim 80 \%$ levels of untreated samples) by rapamycin treatment (Fig. 6b). The levels of non-catalytic subunits of proteasomes, including the 20S subunits Psma1 and Psmb1, the 19S regulatory particle subunit Psmd1, and the $11 \mathrm{~S}$ regulatory particle subunit Psme1, were changed between 1.5- and 2-fold (Fig. 6b), suggesting increase and decrease in proteasome numbers in Tsc1-cko and rapamycin-treated mouse retinas, respectively. However, corresponding changes in protein levels in Tsc1-cko and rapamycin-treated mouse retinas compared with control Tsc1-het and vehicle-treated samples were difficult to detect, except for 35 (Psmb5) and Psmd1 (Fig. 6c, d; Psmb1 and Psme1 protein levels were not examined).

It was shown that a precursor form of immunoproteasome subunits interacts with mTORC1 via Pras40 in prior to its maturation and incorporation into a functional immunoproteasome ${ }^{40}$. Interestingly, mRNA and protein levels of Psmb9/ $/ 31 \mathrm{i}$, an inducible $\beta 1$ isoform in the immunoproteasome ${ }^{41}$, showed the greatest increase in Tsc1-cko mouse retinas and decrease in rapamycin-treated mouse retinas (Fig. 6b-d; Supplementary Fig. 11a). Furthermore, the Psmb9 was also successfully incorporated into $20 \mathrm{~S}$ proteasomes in the Tsc1-cko mouse retina (Fig. 6e), suggesting the formation of functional Psmb9containing immunoproteasomes in the retina. These results suggest that immunoproteasome catalytic subunits might be one that contributes significantly to elevate cellular proteasome activity in Tsc1-cko mouse retinas, where $P s m b 6 / \beta 1$ and Psmb7/ $\beta 2$ were not increased significantly (Fig. 6c, d). However, the other immunoproteasome subunits, Psmb8/ 35 i and Psmb10/ $\beta 2 \mathrm{i}$, were not expressed at significant levels in mouse retinas, although they were increased in E17.5 Tscl ${ }^{f / f_{;}}$Emx1-Cre mouse cerebrum and decreased in the rapamycin-treated adult mouse spleen (Fig. 6b-d; Supplementary Fig. 11b).

Psmb9 is necessary for the developmental acceleration of Tsc1cko mouse retinas. To delineate the roles of Psmb9 in mTORC1promoted retinal development, we generated combinatorial Tsc1cko and Psmb9-ko mice. Deletion of Psmb9 alone did not cause remarkable anatomical changes in embryonic or adult mouse retinas (Supplementary Fig. 12a; results of adult retina are not shown). The numbers of proliferating RPCs and retinal neurons in E14.5 Psmb9-ko mouse retinas were also not greatly different from those in WT and Psmb9-het littermates (Fig. 7a, b; Supplementary Fig. 12a; WT results are not shown). In contrast, the combined loss of Psmb9 and Tsc1 in E14.5 Psmb9-ko;Tsc1-cko mouse retinas significantly decreased the number of Tuj1-positive retinal neurons compared with that observed in Psmb9-het;Tsc1cko littermate mouse retinas (Supplementary Fig. 12a, second row). There were also reduced numbers of BrdU-positive proliferating RPCs and pH3-positive mitotic RPCs in Psmb9-ko;Tsc1cko mouse retinas compared with those in Psmb9-het;Tsc1-cko littermate mouse retinas (Fig. 7b; Supplementary Fig. 12a, third and fourth rows). The populations of BrdU;pH3, BrdU;Otx2, BrdU;Brn3b, and CldU;IdU double-positive cells in Psmb9-ko; Tsc1-cko mouse retinas were also all smaller than those in Psmb9het;Tsc1-cko littermate mouse retinas and indistinguishable from those in Psmb9-het;Tsc1-het and Psmb9-ko;Tsc1-het mouse retinas (Fig. 7a, c), suggesting that Psmb9 is necessary for cell cycle acceleration in Tsc1-deficient RPCs. However, the number of pS6positive retinal cells in Psmb9-ko;Tsc1-cko mouse retinas was not different from that in littermate Psmb9-het;Tsc1-cko mouse retinas (Fig. 7b; Supplementary Fig. 12a), reinforcing the conclusion that Psmb9 acts downstream of or in parallel with mTORC1 activation.

The effects of Psmb9 gene loss on the proliferation of Tsc1deficient RPCs were also tested in vitro. The number of cells in a neurosphere derived from a Tsc1-deficient RPC is larger than that from a Tsc1-het RPC, implicating that Tsc1-deficient RPCs expanded faster than Tsc1-het RPCs during the culture (Supplementary Fig. 12b, c). Not only total number of cells but also the number of BrdU; $\mathrm{pH} 3$ double-positive cell population in a Tsc1-cko;Psmb9-ko retinal neurosphere were decreased in comparison to Tsc1-cko retinal neurospheres (Supplementary Fig. 12b-d), suggesting that the loss of Psmb9 inhibits the expansion of Tsc1-deficient RPC clone by decelerating cell cycle progression.

Despite the deceleration of cell cycle progression of Tsc1-cko mouse RPCs by concomitant loss of Psmb9, the levels of CcnA1, CcnB1, and CcnE1 observed in Psmb9-ko;Tsc1-cko mouse retinas were not fully restored to the levels observed in Psmb9-het;Tsc1het littermate mouse retinas (Fig. $7 \mathrm{~d}, \mathrm{e}$ ). This might be related with the reduced proteasomal activity, especially the immunoproteasome activity, in the retinas. In support of this, the activity of immunoproteasomes, determined by assessing cleavage of the specific substrate Ac-PAL-AMC, was significantly increased in E14.5 Tsc1-cko mouse retinas compared with that in Psmb9-het; Tsc1-het littermate mouse retinas, but was almost undetectable in Psmb9-ko;Tsc1-cko littermate mouse retinas (Fig. 7f). Furthermore, the activity of the caspase-like proteasome $\beta 1 / \beta 1$ i subunit was also decreased significantly in Psmb9-ko;Tsc1-cko mouse retinas relative to Psmb9-het;Tsc1-cko littermate mouse retinas, suggesting that Psmb9 is mainly responsible for the increase in caspase-like proteasome activity in the Tsc1-cko mouse retina. 
a

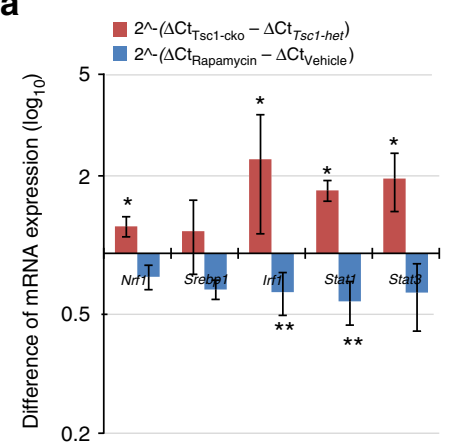

b

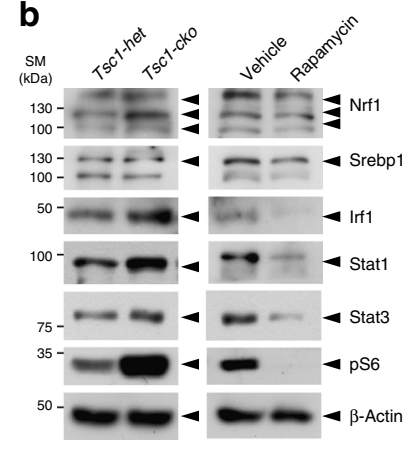

C

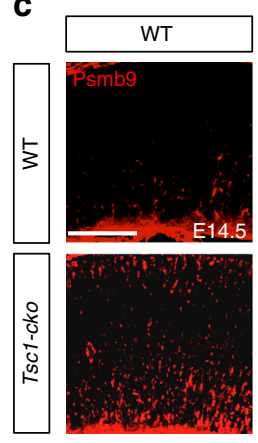

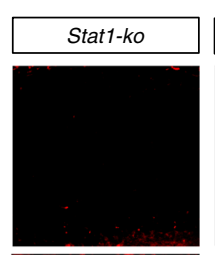

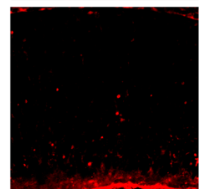

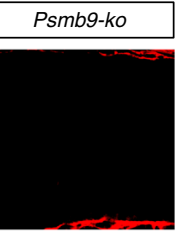

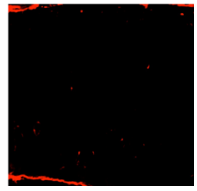

d

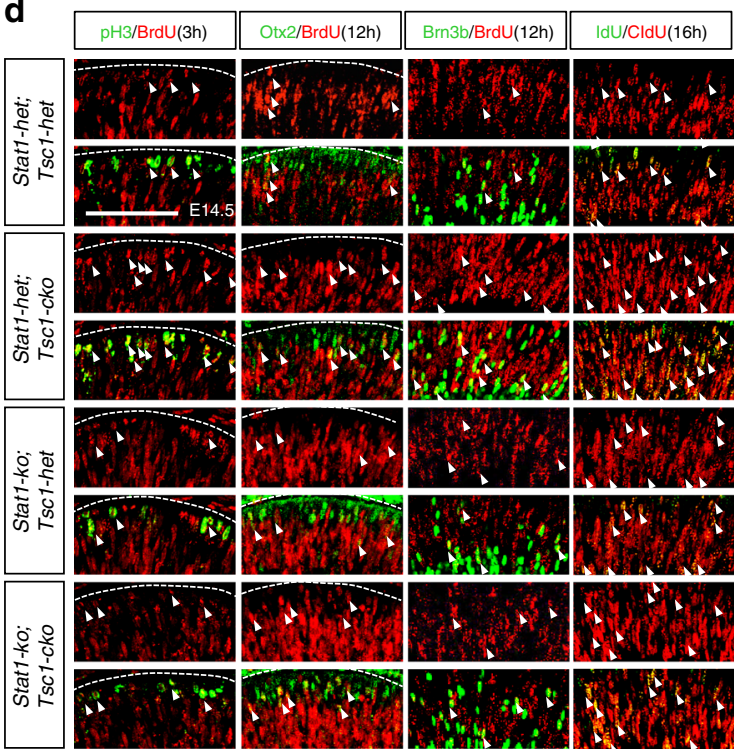

e

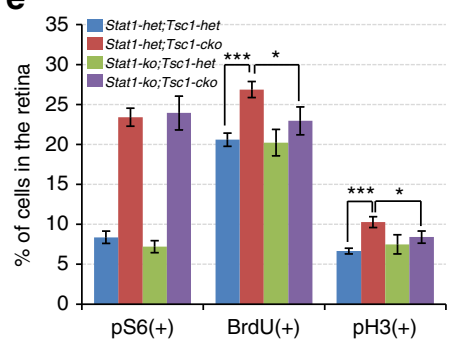

f

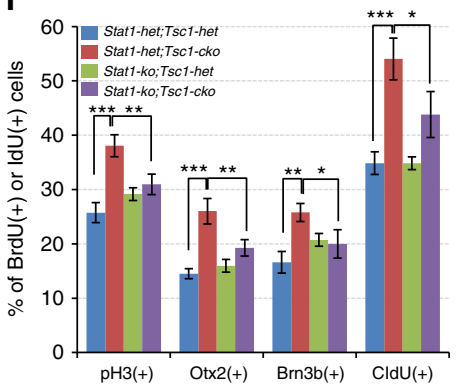

g
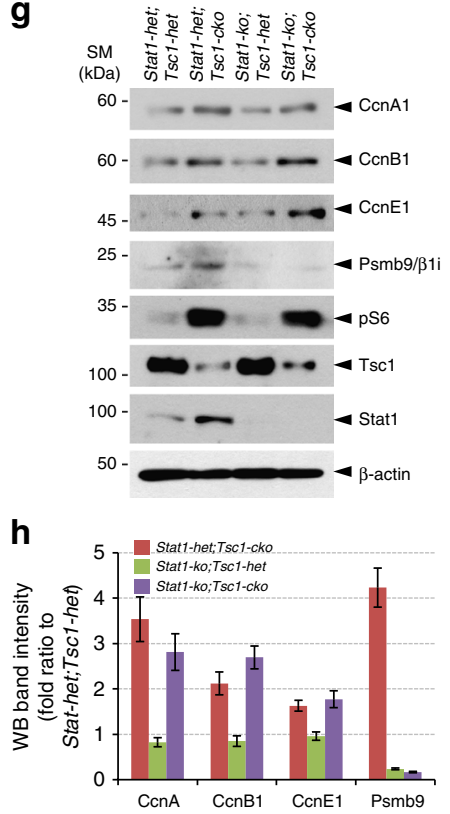

Fig. 8 mTORC1 mediates Stat1 to induce Psmb9 in RPCs. a Relative mRNA levels of transcription factors, which have been known as regulators of proteasome subunit expression $39,42,46$, in E14.5 Tsc1-cko and rapamycin-treated mouse retinas were obtained by comparing RT-qPCR values with those of Tsc1-het and vehicle-treated mouse retinas at the same age. Error bars denote SD $(n=5)$. b The transcription factor proteins in E14.5 mouse retinas were analyzed by WB with corresponding antibodies. Multiple bands of Nrf1 are products of glycosylation ${ }^{62}$. c Distribution of Psmb9 in E14.5 mouse retinas with indicated genotypes was examined by immunostaining with anti-Psmb9 antibody. Scale bar, $100 \mu \mathrm{m}$. d Cell cycle progression of RPCs in E14.5 mouse retinas with combinatorial Tsc1-cko and Psmb9-ko alleles was examined as described in Fig. 2f. Scale bar, $100 \mu$ m. e Average percentage of cells expressing each marker in the retinas was provided in a graph. $\mathbf{f}$ Ratio of BrdU-co-expressing cells to total marker-expressing cells in $\mathbf{d}$ was also shown in a graph. Numbers of mice used are 4 and from 3 independent litters; error bar denotes SD. g Retinal cell lysates (20 $\mu$ g proteins/lane) obtained from E14.5 littermate mouse embryos were analyzed by WB with corresponding antibodies. $\mathbf{h}$ Image pixels of WB bands in each retinal sample were calculated by ImageJ software and relative intensities were obtained by comparing the pixel numbers with those of Stat1-het; Tsc1-het samples. Values are average measurements of 3 independent WB results. Error bars denote SD. P-values are obtained by one-way ANOVA test and shown in the graphs ( ${ }^{\star}<0.05$; $\left.\star \star<0.01 ;{ }^{* \star *}<0.001\right)$

These results implicate that cyclins in Psmb9-ko; Tsc1-cko mouse retinas are synthesized as fast as those in Tsc1-cko mouse retinas, however their proteasomal degradation is less efficient.

To test this hypothesis, we compared the degradation of $\left[{ }^{35} \mathrm{~S}\right]$ labeled CcnB1 in E14.5 Psmb9-ko;Tsc1-cko mouse retinal explants with those in E14.5 WT and Tsc1-cko mouse retinal explants. These experiments showed that the decay of $\left[{ }^{35} \mathrm{~S}\right]$-labeled $\mathrm{CcnB} 1$ and CcnE1 in Psmb9-ko;Tsc1-cko mouse retinal explants was slower than that in Tsc1-cko mouse retinal explants (Fig. 7g-i; Supplementary Fig. 9c). Collectively, these results suggest that activated mTORC1 in Tsc1-deficient mouse RPCs induces Psmb9 and consequently the formation of Psmb9-containing immunoproteasomes, which in turn degrade accumulated cellular substrates, including $\mathrm{CcnB1}$ and $\mathrm{CcnE} 1$, to accelerate cell cycle progression.
mTORC1 acts through Stat1 to induce Psmb9 in mouse RPCs. Psmb9 expression is known to be regulated by various transcription factors, including interferon regulatory factor 1 (Irf1), Stat 1 , and nuclear factor- $\kappa \mathrm{B}(\mathrm{NF}-\kappa \mathrm{B})$, in response to interferon- $\gamma$ $\left(\right.$ IFN- $\gamma$ ) and tumor necrosis factor- $\alpha^{42,43}$. The level of inhibitor of $\mathrm{NF}-\kappa \mathrm{B}$, which is a transcription target of NF- $\kappa \mathrm{B}$ that binds to NF$\kappa \mathrm{B}$ and interferes with its nuclear translocation ${ }^{44}$, was not substantially different between Tsc1-het and Tsc1-cko mouse retinas (data not shown). On the contrary, both mRNA and protein levels of Stat1 and Irf1 in E14.5 Tsc1-cko mouse retinas were increased significantly compared with those in littermate Tsc1-het mouse retinas (Fig. 8a, b). Stat1 has been shown to interact with mTOR $^{45}$ and upregulate Psmb9 gene expression directly or indirectly via $\operatorname{Irf1} 1$ induction ${ }^{46}$. These results therefore suggest that 
the Stat 1 could be a candidate for connecting $\mathrm{mTORC} 1$ activation to Psmb9 induction.

To test this hypothesis, we created Stat1-ko;Tsc1-cko mice in which both Stat1 and Tsc1 were eliminated. Psmb9 expression in E14.5 Stat1-ko;Tsc1-cko mouse retinas was almost undetectable (Fig. 8c, g). Similar to the results obtained in Psmb9-ko mouse retinas, deletion of Stat1 alone did not result in significant changes in mouse retinas (Fig. 8d [third row]-f). In contrast, not only the numbers of CldU;IdU double-labeled cells but also those of mitotic RPCs (pH3-positive) and PMNs (Brn3b-positive and Otx2-positive), which were co-labeled with BrdU for 3 and $12 \mathrm{~h}$, respectively, were decreased significantly in E14.5 Stat1-ko;Tsc1cko mouse retinas compared with Stat1-het;Tsc1-cko littermate mouse retinas (Fig. 8d, f), suggesting that the absence of Stat1 normalizes the cell cycle speed in Tsc1-deficient RPCs.

The levels of CcnA1, CcnB1, and CcnE1 in Stat1-ko;Tsc1-cko mouse retinas were not fully recovered to normal levels (Fig. 8g, h), similar to the results obtained in Psmb9-ko;Tsc1-cko mouse retinas (Fig. 7e). The recovery of regular cell cycle period without the normalization of cyclin protein levels in Psmb9-ko;Tsc1-cko and Stat1-ko;Tsc1-cko mouse retinas might be accounted by the delayed degradation of cyclin proteins, which were synthesized rapidly by elevated mTORC1 activity, in the absence of Psmb9 induction. Collectively, these results suggest that the Stat1-(Irf1)Psmb9 cascade plays an important role in accelerating cell cycle to rapidly expand the Tscl-deficient retinal cell population and develop the Tsc1-cko mouse retina earlier than dictated by the regular developmental schedule.

\section{Discussion}

Our study demonstrates a role for mTORC1 in coordinately regulating the synthesis and degradation of proteins in mouse RPCs. The latter effects of mTORC1 are mediated by upregulation of proteasome subunits, especially the immunoproteasome subunit Psmb9 in the RPCs. The immunoproteasome subunits were also elevated in the Tsc flox/flox; Emx1-Cre mouse embryonic brain and were decreased in the rapamycin-treated adult mouse spleen (Supplementary Fig. 11), suggesting that induction of the immunoproteasome is a common outcome of mTORC1 activation. By elevating the levels of proteasomes-both constitutive proteasomes and immunoproteasomes (Fig. $6 \mathrm{~b}-\mathrm{d}$ ) - mTORC1 might be able to terminate cellular events that are induced by the proteins subjected to proteasomal degradation. However, the degradation of total cellular proteins in Tsc1-cko mouse retinas and rapamycin-treated mouse retinas are not accordingly changed (Supplementary Fig. 10), implicating the mTORC1-sensitive proteasomal degradation is not applied to all proteins but specific targets. We demonstrate C $\mathrm{CnB} 1$ and $\mathrm{C} \mathrm{cnE} 1$ as examples of the proteins that are subject to mTORC1-regulated proteasomal degradation in the developing mouse retina. The cyclins were also synthesized more rapidly in Tsc1-cko mouse retinas potentially through translational activation (Fig. 5f). Therefore, through mTORC1-dependent coordinated synthesis and degradation of cyclin proteins, RPCs might be able to divide without interruptions caused by an insufficient supply or persistent accumulation of cyclins.

An important cellular application of the inducible feature of immunoproteasome is clearing aberrantly accumulated proteins (i.e., antigens) in antigen-presenting cells ${ }^{47}$. The immunoproteasome might also be useful in other cell types in degrading endogenous proteins that accumulate very rapidly and cannot be cleared by constitutive proteasomes alone. Because mTORC1 activity robustly enhances cellular protein synthesis through activation of $\mathrm{S} 6$ and eukaryotic translation initiation factor $4 \mathrm{E}$, it would be incumbent upon cells in which mTORC1 is hyperactive to have a mechanism capable of handling rapidly accumulated proteins. Otherwise, the cell's fate might be altered by these excessive proteins, which could persistently activate their associated cellular events or induce protein stress responses by forming aggregates ${ }^{48}$. Thus, induction of the immunoproteasome could also be an effective strategy for Tsc1-deficient cells to degrade proteins that had rapidly accumulated in the cell.

It should be noted that the role of mTORC1 in proteasomemediated protein degradation is still a matter of debate. Zhang et al. reported that mTORC1 coordinately activates total protein synthesis and degradation. The enhanced protein degradation in Tsc2-ko mouse embryonic fibroblasts and neurons is mediated by Srebp1-Nrf1-dependent upregulation of proteasome subunit gene expression. However, Zhao et al. reported that mTORC1 inhibits proteasomal degradation of a majority of cellular proteins. An inhibitory role of TOR in proteasome was also proposed in another report that shows TOR post-transcriptionally downregulates chaperones involved in the assembly of 19S regulatory particles in yeast and mammals ${ }^{49}$. The $20 \mathrm{~S}$ core particles of immunoproteasomes are capable of forming mature complexes both $19 \mathrm{~S}$ and $11 \mathrm{~S}$ regulatory particles, whereas those of constitutive proteasome associate only with $19 \mathrm{~S}^{50}$. Therefore, the immunoproteasomes comprised of 20S-11S particles might play important roles for protein degradation in the conditions that mTOR inhibits the assembly of $19 \mathrm{~S}$ subunits and consequently decreases the content of mature constitutive proteasomes. Additionally, one caveat of the paper of Zhao et al. is that the inhibitory effects of mTORC1 on proteasome-mediated protein degradation were most applicable to proteins that turn over slowly, not to those with rapid turnover. In support of this idea, the levels of CcnA1, CcnB1, and CcnE1, which among cyclins have relatively short half-lives, were more sensitivity to mTORC1 activity than CcnD1, which has a longer half-life and showed no remarkable differences in levels in response to mTORC1 activity (Fig. 5a-c). By accelerating the turnover of these short-lasting cyclins in RPCs, mTORC1 would be able to shorten the time required to progress to next cell cycle phases and thus the time necessary for cell division (Supplementary Fig. 13).

As we showed in the Tsc1-cko mouse retina, the accumulation of cyclin A, B, and E has also been reported in the Drosophila tsc1 mutant eye disc, and has been proposed as a cause of the hyperproliferation of $t s c 1$ mutant eye disc cells ${ }^{51,52}$. In contrast, similar to Raptor-cko mouse retinas (Fig. 4; Supplementary Fig. 7), raptor mutant fly eyes show diminished proliferation and reduced size ${ }^{53}$, suggesting an evolutionarily conserved role for TORC1 in retinal growth and development by regulating cell proliferation. Therefore, dTORC1-regulated retinal development in Drosophila might be also related to the accelerated turnover of cyclin proteins by proteasomes in addition to their facilitated synthesis. This could be accomplished by constitutive proteasomes, because no immunoproteasome subunit gene has yet been identified in the Drosophila genome.

We have investigated cellular mechanisms involving Stat1, showing that this transcription factor is necessary to induce Psmb9 in the retina as it serves during the cellular response to interferon in the immune system ${ }^{46}$. Stat 1 itself is dispensable for retinal development, although it is necessary for accelerating this development in the context of hyperactivated mTORC1, as is also the case for Psmb9 (Figs. 7 and 8). In contrast, deletion of Stat 3 as well as co-deletion of Stat 3 and $T s c 1$ in mouse RPCs failed to induce any remarkable differences compared with their respective WT and Tsc1-cko mouse retina controls (data not shown), suggesting that Psmb9 induction is a specific activity of Stat1. However, the mechanism of Stat 1 regulation by mTORC1 has been relatively less studied than that of Stat3, which is as a direct target of mTORC1 and supports the proliferation and survival of 
neural stem cells ${ }^{54}$. mTOR has been shown to form a complex with Stat1 in response to lipopolysaccharide or IFN- $\gamma$ and augment Stat1-dependent gene transcription ${ }^{45}$. However, whether mTOR activates Stat 1 activity by directly phosphorylating it or whether the mTOR-Stat1 complex induces Psmb9 in the nucleus remains unclear. Thus, further studies are necessary to delineate the mechanistic connection between mTOR and Stat1 in Psmb9 expression.

Rapamycin is a well-known immunosuppressant that binds to FK506-binding protein, thereby inhibiting calcineurin and subsequent nuclear factor of activated T-cell-dependent production of interleukin- $2^{55}$. Rapamycin also inhibits T-cell activation by interfering with the clonal expansion and antigen-presenting activity of dendritic cells ${ }^{56}$. Our study showed that rapamycin treatment inhibits immunoproteasome $\beta$-subunit expression in the adult mouse spleen (Supplementary Fig. 11a), providing an alternative mechanistic interpretation of rapamycin-induced immune suppression. Because the levels of multiple immunoproteasome subunits were increased in the Tscl-cko mouse brain (Supplementary Fig. 11b), future studies should also investigate the potential involvement of the immunoproteasome in various brain disorders, such as autism and epilepsy, caused by mTORC1 hyperactivation.

\section{Methods}

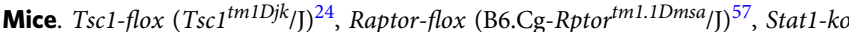
$\left(B 6.129 S(C g)-S_{t a t} t^{t m} 1 D l v / J\right)^{58}$, Stat3-flox (Stat $\left.{ }^{\text {tm2Aki }} / /\right)^{59}$, and R26R (B6.129S4-Gt (ROSA)26Sor $\left.{ }^{\text {tm } 1 S o r} / \mathrm{J}\right)^{25}$ were obtained from Jackson Laboratory, Maine, USA. Psmb9-ko mice (B6.129P2-Psmb $9^{\langle t m 1 S t l>}$ ), which were deposited by Dr. Hajime Hisaeda ${ }^{60}$, were obtained from RIKEN BioResource Center, Tsukuba, Japan. Chx10-Cre-GFP (Chx10-Cre) mice were obtained by a generous gift from Dr. Connie Cepko ${ }^{23}$. Experiments using the mice were carried out according to the guidance of KAIST IACUC-2013-54.

Antibodies and immunostaining. Mouse embryos were collected from pregnant mice, which were injected with $\mathrm{BrdU}(5 \mathrm{mg} / \mathrm{kg})$ for the indicated time periods in prior to sample preparation. To inhibit mTORC1 activity in the mouse embryos, rapamycin stock solution $(10 \mathrm{mg} / \mathrm{ml}$ ethanol) was diluted in vehicle $(5 \%$ polyethylene glycol and 5\% Tween 80 in phosphate-buffered saline (PBS)) for the injection into the intraperitoneal space of the pregnant mice as the indicated schedule in the figures. The embryos were isolated and then fixed in PBS (pH 7.4) containing $4 \%$ paraformaldehyde (PFA) for $3 \mathrm{~h}$ at room temperature. Alternatively, eyes were isolated from the post-natal mice, which were perfused $4 \% \mathrm{PFA} / \mathrm{PBS}$. The eyes with needle punctures were then incubated in $4 \% \mathrm{PFA} / \mathrm{PBS}$ solution for $16 \mathrm{~h}$ at $4{ }^{\circ} \mathrm{C}$. The embryos and eyes were transferred to $20 \%$ sucrose/PBS solution until they sank down before subsequent freezing in OCT medium. Immunostaining for cryosections of the embryos and post-natal mouse eyes were performed as reported previously ${ }^{14}$. List of antibodies used for this work were provided in Supplementary Table 1.

Fluorescence-activated cell sorting (FACS) analysis. P14 Tsc1-het and Tsc1-cko adult mouse eyes were dissected and isolated individual retinal cells as described previously ${ }^{61}$. In brief, the cells were incubated in Hank's balanced salt solution (HBSS) containing 4',6-diamidino-2-phenylindole (DAPI; $1 \mu \mathrm{g} / \mathrm{ml}$, final) for 10 min, and fluorescent emitted by DAPI-stained dead cells were detected at $405 \mathrm{~nm}$ excitation and $452 \mathrm{~nm}$ emission using Beckman Courter Flow Cytometer. Relative sizes of DAPI-negative retinal cells were then determined by measuring forward scatter. Relative sizes of BPs were determined by comparing Chx10::GFP-positive cells among DAPI-negative retinal cells analyzed at $495 \mathrm{~nm}$ excitation and $519 \mathrm{~nm}$ emission.

Real-time quantitative PCR. To compare mRNA levels of cell cycle regulators and proteasome subunits, first their cDNA was obtained by reverse transcription with oligo-dT and M-MLV reverse transcriptase (Bioneer). Real-time quantitative PCR (RT-qPCR) was then performed with CFX96 Real-Time System (Bio-Rad) by using iTaq Universal SYBR Green Supermix (Bio-Rad). The RT-qPCR conditions were as follows: $95^{\circ} \mathrm{C}$ for $30 \mathrm{~s}$, followed by 40 cycles at $95^{\circ} \mathrm{C}$ for $10 \mathrm{~s}$ and $60^{\circ} \mathrm{C}$ for $30 \mathrm{~s}$, and melt curve stage were $95^{\circ} \mathrm{C}$ for $15 \mathrm{~s}, 60^{\circ} \mathrm{C}$ for $1 \mathrm{~min}$, and $95^{\circ} \mathrm{C}$ for $15 \mathrm{~s}$. The RTqPCR was performed with three biological replications, and relative transcript levels of gene of interest were calculated according to the $2^{-\Delta \Delta \mathrm{Ct}}$ method against $\beta$ actin mRNA. Primer sequences used for the RT-qPCR are provided in Supplemental Table 2. $\left.{ }^{35} \mathrm{~S}\right]-$ methionine pulse labeling and chasing in retinal explants. E13.5 retinal quadrants were incubated in N2-supplemented Dulbecco's modified Eagle's medium (DMEM; Gibco) with 15\% fetal bovine serum (FBS) and amino acids but without methionine for $3 \mathrm{~h}$. The $\left[{ }^{35} \mathrm{~S}\right]$-labeled methionine $\left(\left[{ }^{35} \mathrm{~S}\right]-\mathrm{Met}, 250 \mu \mathrm{Ci} / \mathrm{ml}\right)$ was applied to the retinal explants for $3 \mathrm{~h}$. The retinal explants were washed twice with PBS and then added with growth medium containing unlabeled Met $(1 \mathrm{mM}$, final concentration) in the presence and absence of CHX $(10 \mu \mathrm{g} / \mathrm{ml})$. Retinal explants were obtained at time points indicated in the figures, and lysed in a cell lysis buffer (10 mM Tris, $150 \mathrm{mM} \mathrm{NaCl}, 1 \%$ NP40, $1 \%$ Triton X-100, and protease inhibitor cocktail [Roche]). Retinal lysates including $0.5 \mathrm{mg}$ proteins were incubated with $1 \mu \mathrm{g}$ anti-CcnB1 or anti-CcnE1 antibodies at $4^{\circ} \mathrm{C}$ for $3 \mathrm{~h}$, and then added with $20 \mu \mathrm{l}$ (wet volume) protein G-sepharose for $2 \mathrm{~h}$ at $4^{\circ} \mathrm{C}$. The immune complexes were centrifuged and washed three times with the cell lysis buffer and twice with the cell lysis buffer with $1 \mathrm{M} \mathrm{NaCl}$. Proteins in the immunoprecipitates were eluted in $20 \mu \mathrm{l} 2 \times$ SDS sample buffer for 10\% SDS-polyacrylamide gel electrophoresis. The radioactive signal in the gel was then visualized in BAS-7000 (Fuji).

$20 S$ proteasome purification and protease activity assay. $20 \mathrm{~S}$ proteasomes were purified from retinal cell lysate in a lysis buffer containing $25 \mathrm{mM}$ HEPES (pH 7.5), 10\% glycerol, $5 \mathrm{mM} \mathrm{MgCl}_{2}, 1 \mathrm{mM} \mathrm{ATP}$, and $1 \mathrm{mM}$ dithiothreitol by the Proteasome Purification Kit (Enzo Life Sciences BWL-PW9005) following the manufacturer's guide. Immune complex of $20 \mathrm{~S}$ proteasomes were incubated with the fluorogenic substrate Z-Leu-Leu-GluAMC (Enzo, BML-ZW9345), Boc-Leu-Arg-Arg-AMC (Enzo, BML-BW8515), LeuLeu-Val-Tyr-AMC (Enzo,BML-P802), or Ac-Pro-Ala-Lys-AMC (Boston Biochem Inc.) to measure the fluorescence emitted by cleaved peptide substrates using fluorescence spectroscopy (Tecan Infinite M200) at excitation and emission wavelengths of 380 and $460 \mathrm{~nm}$, respectively, according to the manufacturer's instruction.

RPC neurosphere culture. The eyes of E13.5 mouse embryos were enucleated and the retina was dissected out in HBSS with care to avoid contamination from other ocular tissues. Retinas were transferred into a dissociation solution (DMEM containing $0.1 \%$ Trypsin and DNAse I $\left(100 \mu \mathrm{g} / \mathrm{ml}\right.$; Sigma) and incubated at $37^{\circ} \mathrm{C}$ in $\mathrm{CO}_{2}$ incubator for $30 \mathrm{~min}$. The retinas were mechanically dissociated into small aggregates and cultured in DMEM containing 5\% FBS for 1 day. The aggregates were then dissociated into single cells using Acumax (Sigma) and grown to form colonies in $0.9 \%(\mathrm{w} / \mathrm{v})$ methylcellulose matrix (DMEM/F12 containing $\mathrm{N} 2$ supplement (GIBCO), B27(GIBCO), $2 \mathrm{mM}$ L-glutamine, $100 \mathrm{U} / \mathrm{ml}$ penicillin, and $100 \mu \mathrm{g} / \mathrm{ml}$ streptomycin) supplemented with fibroblast growth factor $2(10 \mathrm{ng} /$ $\mathrm{ml}$; R\&D Systems) and epidermal growth factor (20 ng/ml; Sigma).

Ubiquitination assay. The retinas were chopped into small pieces and lysed in a buffer (2\% SDS, $150 \mathrm{mM} \mathrm{NaCl}, 10 \mathrm{mM}$ Tris-Cl, $\mathrm{pH}$ 8.0) with protease inhibitor (Calbiochem) and protein phosphatase inhibitor (Sigma) cocktails for $5 \mathrm{~min}$. The cell lysates were boiled for $10 \mathrm{~min}$ and then cooled down at room temperature in prior to sonication on ice. Ten volumes of a dilution buffer ( $10 \mathrm{mM}$ Tris-Cl, $\mathrm{pH}$ 8.0, $150 \mathrm{mM} \mathrm{NaCl}, 2 \mathrm{mM}$ EDTA, and $1 \%$ Triton) were added to the cell lysates before the centrifugation at $20000 \times g$ for $30 \mathrm{~min}$. Lysates including $1 \mathrm{mg}$ proteins were subjected to the immunoprecipitation with antibodies against proteins of interest for subsequent western blot detection of ubiquitinated proteins with mouse anti-ubiquitin antibody.

Statistical analyses. Statistical analysis has been performed with SAS software (version 9.4). Group comparison was done using two-sample Student's $t$-test and analysis of variance test, unless it was specified in figure legends. A difference was considered significant at $P<0.05$.

Data availability. The authors declare that all relevant data of this study are included within the article and its Supplementary Information. The data are also available from the authors upon reasonable request.

Received: 30 May 2017 Accepted: 26 April 2018

Published online: 27 June 2018

\section{References}

1. Cepko, C. Intrinsically different retinal progenitor cells produce specific types of progeny. Nat. Rev. Neurosci. 15, 615-627 (2014).

2. Bjornsson, C. S., Apostolopoulou, M., Tian, Y. \& Temple, S. It takes a village: constructing the neurogenic niche. Dev. Cell 32, 435-446 (2015).

3. Alexiades, M. R. \& Cepko, C. Quantitative analysis of proliferation and cell cycle length during development of the rat retina. Dev. Dyn. 205, 293-307 (1996). 
4. Sicinski, P. et al. Cyclin D1 provides a link between development and oncogenesis in the retina and breast. Cell 82, 621-630 (1995).

5. Das, G., Choi, Y., Sicinski, P. \& Levine, E. M. Cyclin D1 fine-tunes the neurogenic output of embryonic retinal progenitor cells. Neural Dev. 4, 15 (2009).

6. Das, G., Clark, A. M. \& Levine, E. M. Cyclin D1 inactivation extends proliferation and alters histogenesis in the postnatal mouse retina. Dev. Dyn. 241, 941-952 (2012).

7. Geng, Y. et al. Deletion of the p27Kip1 gene restores normal development in cyclin D1-deficient mice. Proc. Natl Acad. Sci. USA 98, 194-199 (2001).

8. Agathocleous, M. \& Harris, W. A. From progenitors to differentiated cells in the vertebrate retina. Ann. Rev. Cell Dev. Biol. 25, 45-69 (2009).

9. Cayouette, M., Barres, B. A. \& Raff, M. Importance of intrinsic mechanisms in cell fate decisions in the developing rat retina. Neuron 40, 897-904 (2003).

10. Kim, J. et al. GDF11 controls the timing of progenitor cell competence in developing retina. Science 308, 1927-1930 (2005).

11. Wang, Y. P. et al. Development of normal retinal organization depends on Sonic hedgehog signaling from ganglion cells. Nat. Neurosci. 5, 831-832 (2002).

12. Lamba, D., Karl, M. \& Reh, T. Neural regeneration and cell replacement: a view from the eye. Cell Stem Cell 2, 538-5492 (2008).

13. Bateman, J. M. \& McNeill, H. Temporal control of differentiation by the insulin receptor/tor pathway in Drosophila. Cell 119, 87-96 (2004).

14. Jo, H. S., Kang, K. H., Joe, C. O. \& Kim, J. W. Pten coordinates retinal neurogenesis by regulating Notch signalling. EMBO J. 31, 817-828 (2012).

15. Cantrup, R. et al. Cell-type specific roles for PTEN in establishing a functional retinal architecture. PLoS ONE 7, e32795 (2012).

16. Sakagami, K., Chen, B., Nusinowitz, S., Wu, H. \& Yang, X. J. PTEN regulates retinal interneuron morphogenesis and synaptic layer formation. Mol. Cell Neursci 49, 171-183 (2012).

17. Laplante, M. \& Sabatini, D. M. mTOR signaling in growth control and disease. Cell 149, 274-293 (2012).

18. Garami, A. et al. Insulin activation of Rheb, a mediator of mTOR/S6K/4E-BP signaling, is inhibited by TSC1 and 2. Mol. Cell 11, 1457-1466 (2003).

19. Zhang, Y. et al. Rheb is a direct target of the tuberous sclerosis tumour suppressor proteins. Nat. Cell Biol. 5, 578-581 (2003).

20. Bar-Peled, L. \& Sabatini, D. M. Regulation of mTORC1 by amino acids. Trends Cell Biol. 24, 400-406 (2014).

21. Costa-Mattioli, M. \& Monteggia, L. M. mTOR complexes in neurodevelopmental and neuropsychiatric disorders. Nat. Neurosci. 16, 1537-1543 (2013).

22. Love, N. K., Keshavan, N., Lewis, R., Harris, W. A. \& Agathocleous, M. A nutrient-sensitive restriction point is active during retinal progenitor cell differentiation. Development 141, 697-706 (2014)

23. Rowan, S. \& Cepko, C. L. Genetic analysis of the homeodomain transcription factor Chx10 in the retina using a novel multifunctional BAC transgenic mouse reporter. Dev. Biol. 271, 388-402 (2004).

24. Kwiatkowski, D. J. et al. A mouse model of TSC1 reveals sex-dependent lethality from liver hemangiomas, and up-regulation of p70S6 kinase activity in Tsc1 null cells. Hum. Mol. Genet. 11, 525-534 (2002).

25. Soriano, P. Generalized lacZ expression with the ROSA26 Cre reporter strain. Nat. Genet. 21, 70-71 (1999).

26. Mori, M., Metzger, D., Garnier, J. M., Chambon, P. \& Mark, M. Site-specific somatic mutagenesis in the retinal pigment epithelium. Invest. Ophthal. Vis. Sci. 43, 1384-1388 (2002).

27. Brown, E. J. et al. A mammalian protein targeted by G1-arresting rapamycinreceptor complex. Nature 369, 756-758 (1994).

28. Sabatini, D. M., Erdjument-Bromage, H., Lui, M., Tempst, P. \& Snyder, S. H. RAFT1: a mammalian protein that binds to FKBP12 in a rapamycindependent fashion and is homologous to yeast TORs. Cell 78, 35-43 (1994).

29. Kim, D. H. et al. mTOR interacts with raptor to form a nutrient-sensitive complex that signals to the cell growth machinery. Cell 110, 163-175 (2002).

30. Sarbassov, D. D. et al. Rictor, a novel binding partner of mTOR, defines a rapamycin-insensitive and raptor-independent pathway that regulates the cytoskeleton. Curr. Biol. 14, 1296-1302 (2004).

31. Sullivan, M. \& Morgan, D. O. Finishing mitosis, one step at a time. Nat. Rev. Mol. Cell Biol. 8, 894-903 (2007).

32. Murray, A. W. Recycling the cell cycle: cyclins revisited. Cell 116, 221-234 (2004).

33. Satyanarayana, A. \& Kaldis, P. Mammalian cell-cycle regulation: several Cdks, numerous cyclins and diverse compensatory mechanisms. Oncogene 28, 2925-2939 (2009).

34. Fung, T. K. \& Poon, R. Y. A roller coaster ride with the mitotic cyclins. Semin. Cell Dev. Biol. 16, 335-342 (2005).

35. Romero-Pozuelo, J., Demetriades, C., Schroeder, P. \& Teleman, A. A. CycD/ Cdk4 and discontinuities in Dpp signaling activate TORC1 in the Drosophila wing disc. Dev. Cell 42, 376-387 (2017).
36. Dyer, M. A. \& Cepko, C. L. p27Kip1 and p57Kip2 regulate proliferation in distinct retinal progenitor cell populations. J. Neurosci. 21, 4259-4271 (2001).

37. Ma, X. M. \& Blenis, J. Molecular mechanisms of mTOR-mediated translational control. Nat. Rev. Mol. Cell Biol. 10, 307-318 (2009).

38. Frankland-Searby, S. \& Bhaumik, S. R. The $26 \mathrm{~S}$ proteasome complex: an attractive target for cancer therapy. Biochim. Biophys. Acta 1825, 64-76 (2012).

39. Zhang, Y. et al. Coordinated regulation of protein synthesis and degradation by mTORC1. Nature 513, 440-443 (2014)

40. Yun, Y. S. et al. mTORC1 coordinates protein synthesis and immunoproteasome formation via PRAS40 to prevent accumulation of protein stress. Mol. Cell 61, 625-639 (2016).

41. Kelly, A. et al. Second proteasome-related gene in the human MHC class II region. Nature 353, 667-668 (1991).

42. White, L. C. et al. Regulation of LMP2 and TAP1 genes by IRF-1 explains the paucity of CD8+ T cells in IRF-1-/- mice. Immunity 5, 365-376 (1996).

43. Wright, K. L. et al. Coordinate regulation of the human TAP1 and LMP2 genes from a shared bidirectional promoter. J. Exp. Med. 181, 1459-1471 (1995).

44. Karin, M., Yamamoto, Y. \& Wang, Q. M. The IKK NF-kappa B system: a treasure trove for drug development. Nat. Rev. Drug Discov. 3, 17-26 (2004).

45. Kristof, A. S., Marks-Konczalik, J., Billings, E. \& Moss, J. Stimulation of signal transducer and activator of transcription-1 (STAT1)-dependent gene transcription by lipopolysaccharide and interferon-gamma is regulated by mammalian target of rapamycin. J. Biol. Chem. 278, 33637-33644 (2003).

46. Chatterjee-Kishore, M., Wright, K. L., Ting, J. P. \& Stark, G. R. How Stat mediates constitutive gene expression: a complex of unphosphorylated Stat and IRF1 supports transcription of the LMP2 gene. EMBO J. 19, 4111-4122 (2000).

47. Basler, M., Kirk, C. J. \& Groettrup, M. The immunoproteasome in antigen processing and other immunological functions. Curr. Opin. Immunol. 25, 74-80 (2013).

48. Ozcan, U. et al. Loss of the tuberous sclerosis complex tumor suppressors triggers the unfolded protein response to regulate insulin signaling and apoptosis. Mol. Cell 29, 541-551 (2008).

49. Rousseau, A. \& Bertolotti, A. An evolutionarily conserved pathway controls proteasome homeostasis. Nature 536, 184-189 (2016).

50. McCarthy, M. K. \& Weinberg, J. B. The immunoproteasome and viral infection: a complex regulator of inflammation. Front. Microbiol. 6, 21 (2015)

51. Potter, C. J., Huang, H. \& Xu, T. Drosophila Tsc1 functions with Tsc2 to antagonize insulin signaling in regulating cell growth, cell proliferation, and organ size. Cell 105, 357-368 (2001).

52. Tapon, N., Ito, N., Dickson, B. J., Treisman, J. E. \& Hariharan, I. K. The Drosophila tuberous sclerosis complex gene homologs restrict cell growth and cell proliferation. Cell 105, 345-355 (2001).

53. Resnik-Docampo, M. \& de Celis, J. F. MAP4K3 is a component of the TORC1 signalling complex that modulates cell growth and viability in Drosophila melanogaster. PLoS ONE 6, e14528 (2011).

54. Androutsellis-Theotokis, A. et al. Notch signalling regulates stem cell numbers in vitro and in vivo. Nature 442, 823-826 (2006).

55. Abraham, R. T. \& Wiederrecht, G. J. Immunopharmacology of rapamycin. Ann. Rev. Immunol. 14, 483-510 (1996).

56. Matsue, H. et al. Contrasting impacts of immunosuppressive agents (rapamycin, FK506, cyclosporin A, and dexamethasone) on bidirectional dendritic cell-T cell interaction during antigen presentation. J. Immunol. 169, 3555-3564 (2002)

57. Sengupta, S., Peterson, T. R., Laplante, M., Oh, S. \& Sabatini, D. M. mTORC1 controls fasting-induced ketogenesis and its modulation by ageing. Nature 468, 1100-1104 (2010).

58. Durbin, J. E., Hackenmiller, R., Simon, M. C. \& Levy, D. E. Targeted disruption of the mouse Stat1 gene results in compromised innate immunity to viral disease. Cell 84, 443-450 (1996).

59. Takeda, K. et al. Stat3 activation is responsible for IL-6-dependent T cell proliferation through preventing apoptosis: generation and characterization of T cell-specific Stat3-deficient mice. J. Immunol. 161, 4652-4660 (1998).

60. Van Kaer, L. et al. Altered peptidase and viral-specific T cell response in LMP2 mutant mice. Immunity 1, 533-541 (1994).

61. Kim, Y. et al. The LIM protein complex establishes a retinal circuitry of visual adaptation by regulating Pax6 alpha-enhancer activity. eLife 6, e21303 (2017).

62. Zhang, Y., Lucocq, J. M., Yamamoto, M., \& Hayes, J. D. The NHB1 (Nterminal homology box 1) sequence in transcription factor Nrf1 is required to anchor it to the endoplasmic reticulum and also to enable its asparagineglycosylation. Biochem. J. 408, 161-172 (2007).

\section{Acknowledgements}

This work was supported by the National Research Foundation of Korea (NRF) grants (2009-00424; 2013M3C7A1056566; and 2017R1A2B3002862) funded by the Korean Ministry of Science, ICT, and Future Planning (MSIP) 


\section{Author contributions}

J.-H.C., H.S.J., and J.W.K. wrote the manusucript; J.-H.C., H.S.J., S.L., H.-T.K., K.W.L., K. H.M., T.H., S.S.K., Y.K., and E.J.L. designed and performed the experiments and analyzed the data; C.O.J., and J.W.K. supervised the project.

\section{Additional information}

Supplementary Information accompanies this paper at https://doi.org/10.1038/s41467018-04774-9.

Competing interests: The authors declare no competing interests.

Reprints and permission information is available online at http://npg.nature.com/ reprintsandpermissions/

Publisher's note: Springer Nature remains neutral with regard to jurisdictional claims in published maps and institutional affiliations. cc (i) Open Access This article is licensed under a Creative Commons Attribution 4.0 International License, which permits use, sharing, adaptation, distribution and reproduction in any medium or format, as long as you give appropriate credit to the original author(s) and the source, provide a link to the Creative Commons license, and indicate if changes were made. The images or other third party material in this article are included in the article's Creative Commons license, unless indicated otherwise in a credit line to the material. If material is not included in the article's Creative Commons license and your intended use is not permitted by statutory regulation or exceeds the permitted use, you will need to obtain permission directly from the copyright holder. To view a copy of this license, visit http://creativecommons.org/ licenses/by/4.0/.

(C) The Author(s) 2018 